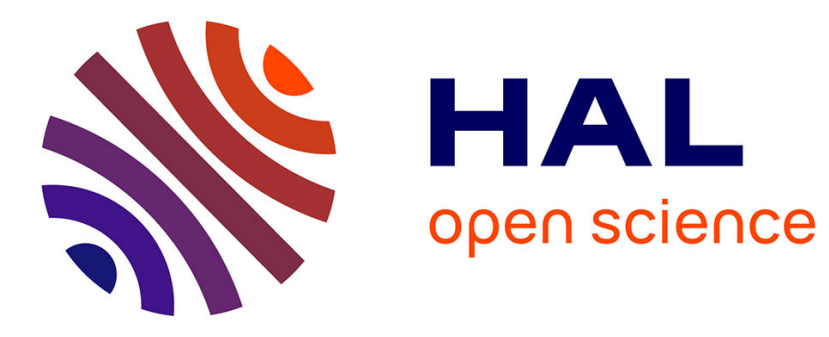

\title{
Homogenization of periodic systems with large potentials
}

\author{
Grégoire Allaire, Yves Capdeboscq, Andrey Piatnitski, Vincent Siess, \\ Muthusamy Vanninathan
}

\section{- To cite this version:}

Grégoire Allaire, Yves Capdeboscq, Andrey Piatnitski, Vincent Siess, Muthusamy Vanninathan. Homogenization of periodic systems with large potentials. Archive for Rational Mechanics and Analysis, 2004, 174 (2), pp.179-220. 10.1007/s00205-004-0332-7 . hal-02083813

\section{HAL Id: hal-02083813 \\ https://hal.science/hal-02083813}

Submitted on 4 Aug 2020

HAL is a multi-disciplinary open access archive for the deposit and dissemination of scientific research documents, whether they are published or not. The documents may come from teaching and research institutions in France or abroad, or from public or private research centers.
L'archive ouverte pluridisciplinaire HAL, est destinée au dépôt et à la diffusion de documents scientifiques de niveau recherche, publiés ou non, émanant des établissements d'enseignement et de recherche français ou étrangers, des laboratoires publics ou privés. 


\title{
Homogenization of periodic systems with large potentials
}

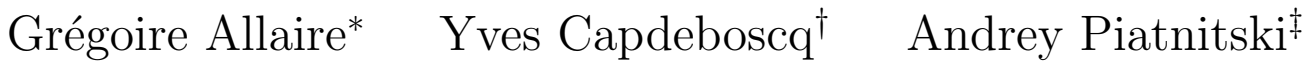 \\ Vincent Siess ${ }^{\S} \quad$ M. Vanninathan
}

This is a post-peer-review, pre-copyedit version of an article published in Archive for Rational Mechanics and Analysis. The final authenticated version is available online at: doi:10.1007/s00205-004-0332-7

\begin{abstract}
We consider the homogenization of a system of second-order equations with a large potential in a periodic medium. Denoting by $\epsilon$ the period, the potential is scaled as $\epsilon^{-2}$. Under a generic assumption on the spectral properties of the cell problem, we prove that the solution can be factorized as the product of a fast oscillating cell eigenfunction and of a slowly varying solution of a scalar second-order equation. This result applies to various types of equations such as parabolic, hyperbolic or eigenvalue problems, as well as fourth-order plate equation. We also prove that for well-prepared initial data concentrating at the bottom of a Bloch band the resulting homogenized tensor depends on
\end{abstract}

\footnotetext{
*Centre de Mathématiques Appliquées, Ecole Polytechnique, 91128 Palaiseau, France - gregoire.allaire@polytechnique.fr

†Département de Mathématiques, INSA Rennes

yves.capdeboscq@insa-rennes.fr

${ }^{\ddagger}$ Narvik Institute of Technology, HiN, P.O.Box 385, 8505, Narvik, Norway and P.N.Lebedev Physical Institute RAS, Leninski prospect 53, Moscow 117333 Russia andrey@sci.lebedev.ru

${ }^{\S}$ CEA Saclay, DEN/DM2S, $91191 \quad$ Gif-sur-Yvette, France siess@soleil.serma.cea.fr

IIISc-TIFR Mathematics Programme, TIFR Center, P.O. Box 1234, Bangalore 560012, India — vanni@math.tifrbng.res.in
} 
the chosen Bloch band. Our method is based on a combination of classical homogenization techniques (two-scale convergence and suitable oscillating test functions) and of Bloch waves decomposition.

\section{Introduction}

We study the homogenization of evolution problems for a singularly perturbed second order elliptic system with periodically oscillating coefficients. To fix ideas, let us consider the following parabolic problem

$$
\begin{cases}\frac{\partial u_{\epsilon}}{\partial t}-\operatorname{div}\left(A\left(\frac{x}{\epsilon}\right) \nabla u_{\epsilon}\right)+\left(\epsilon^{-2} c\left(\frac{x}{\epsilon}\right)+d\left(x, \frac{x}{\epsilon}\right)\right) u_{\epsilon}=0 & \text { in } \Omega \times(0, T), \\ u_{\epsilon}=0 & \text { on } \partial \Omega \times(0, T), \\ u_{\epsilon}(t=0, x)=u_{\epsilon}^{0}(x) & \text { in } \Omega,\end{cases}
$$

where $\Omega \subset \mathbb{R}^{N}$ is an open set and $T>0$ a final time. The unknown $u_{\epsilon}(t, x)$ is a vector-valued function from $\Omega \times(0, T)$ into $\mathbb{R}^{K}$. The coefficients $A(y), c(y)$ and $d(x, y)$ are real and bounded functions defined for $x \in \Omega$ and $y \in \mathbb{T}^{N}$ (the unit torus). Furthermore, the tensor $A(y)$ is symmetric, uniformly positive definite, while $c(y)$ and $d(x, y)$ are symmetric with no positivity assumption. The parabolic equation (11) is just an example: other evolution problems of interest covered by this paper are the wave equation, parabolic fourth-order equations, or spectral problems. A generalization to the Schrödinger equation is the topic of another work [9]. The scalar case of (1) (i.e. $K=1$ and $u_{\epsilon}$ is a real-valued function) is well understood (see e.g. [4], [7], [8], [21], [28]) and the goal of this paper is to solve the case of systems of several coupled equations. However, the method, as well as some results, are very different in the system case. In order to convince the reader, we first describe the main results and ideas of proof in the scalar case.

For $K=1$ introduce the first eigencouple of the spectral cell problem

$$
-\operatorname{div}_{y}\left(A(y) \nabla_{y} \psi_{1}\right)+c(y) \psi_{1}=\lambda_{1} \psi_{1} \quad \text { in } \mathbb{T}^{N},
$$

which, by the Krein-Rutman theorem, is simple and satisfies $\psi_{1}(y)>0$ in $\mathbb{T}^{N}$. One can interpret physically the first eigenvalue $\lambda_{1}$ as a measure of the balance between the diffusion and potential terms. Since $\psi_{1}$ does not vanish, the unknown can be changed by writing a so-called factorization principle

$$
v_{\epsilon}(t, x)=e^{\frac{\lambda_{1} t}{\epsilon^{2}}} \frac{u_{\epsilon}(t, x)}{\psi_{1}\left(\frac{x}{\epsilon}\right)},
$$


and one check easily after some algebra that the new unknown $v_{\epsilon}$ is a solution of a simpler equation

$$
\begin{cases}\psi_{1}^{2}\left(\frac{x}{\epsilon}\right) \frac{\partial v_{\epsilon}}{\partial t}-\operatorname{div}\left(\left(\psi_{1}^{2} A\right)\left(\frac{x}{\epsilon}\right) \nabla v_{\epsilon}\right)+\left(\psi_{1}^{2} d\right)\left(x, \frac{x}{\epsilon}\right) v_{\epsilon}=0 & \text { in } \Omega \times(0, T), \\ v_{\epsilon}=0 & \text { on } \partial \Omega \times(0, T), \\ v_{\epsilon}(t=0, x)=\frac{u_{\epsilon}^{0}(x)}{\psi_{1}\left(\frac{x}{\epsilon}\right)} & \text { in } \Omega .\end{cases}
$$

The new parabolic equation (4) is simple to homogenize since it does not contain any singularly perturbed term, and we thus obtain the following result.

Theorem 1.1 Assume that (1) is a scalar problem $(K=1)$. Then, $v_{\epsilon}$, defined by (3), converges weakly in $L^{2}\left((0, T) ; H_{0}^{1}(\Omega)\right)$ to the solution $v$ of the following homogenized problem

$$
\begin{cases}\frac{\partial v}{\partial t}-\operatorname{div}\left(A^{*} \nabla v\right)+d^{*}(x) v=0 & \text { in } \Omega \times(0, T) \\ v=0 & \text { on } \partial \Omega \times(0, T) \\ v(t=0, x)=v^{0}(x) & \text { in } \Omega\end{cases}
$$

where $A^{*}$ is a constant homogenized tensor and $d^{*}(x)$ a homogenized coefficient.

It is clear from the above brief summary of the scalar case that the main idea, namely the factorization principle (3), does not usually work in the case of systems, i.e. $K>1$. Indeed, in general there is no maximum principle, and therefore no Krein-Rutman theorem, for systems. Thus, $\psi_{1}$ may change sign and the change of unknowns (3) is meaningless because $v_{\epsilon}$ blows up at some points (see however [4] for a special system for which the maximum principle holds true). Even if we perform a formal computation by assuming that (3) is valid, the system satisfied by $v_{\epsilon}$ has not a simple structure and it is not clear that it admits a homogenized limit, and even so, there is no reason why the homogenized tensor should be coercive.

In order to homogenize (1) in the system case, our main new idea is to use Bloch wave theory. Under a generic simplicity assumption for the first eigenvalue and a non-degenerate quadratic behavior near its minimum (see (9) ) we obtain a result similar to Theorem 1.1 (see Theorem 3.2 for details). The two main features are that the homogenized equation is always 
scalar and that the cell problem must sometimes be shifted, namely the usual periodicity condition in (2) has to be replaced by a Bloch periodicity condition. Our analysis applies not only to the parabolic problem (1) but also to the corresponding spectral problem and hyperbolic system. Section 2 contains our notations, a brief review of Bloch wave theory and our main assumption. Our main results are stated in Section 3 while the proofs are distributed in Sections 4, 5 and 6.

In Section 7 we also obtain new homogenization results for some specific well-prepared initial data (assuming that $\Omega=\mathbb{R}^{N}$ ). More precisely, recall that Bloch wave theory introduces the notion of Bloch bands, corresponding to the range of cell eigenvalues or, in physical terms, to energy levels of Fermi surfaces. Theorem 1.1 is concerned with the first Bloch band (or ground state). If we assume that the initial data $u_{\epsilon}^{0}$ is concentrating at the bottom of a higher level Bloch band (see Section 7 for a precise statement), we obtain a convergence result similar to Theorem 1.1 but with a different homogenized tensor (depending on the level of the chosen Bloch band). Even in the scalar case this result is new. In the context of Schrödinger equation it is known as an effective mass theorem (see e.g. [22], [24], 25]). The fact that the homogenized tensor depends on the initial data is very striking in homogenization theory since usually effective properties are proved to be intrinsic in the sense that they do not depend on the domain, the applied forces or source terms, and the initial data.

In Section 8 we show that under a new assumption on the first Bloch eigenvalue a different homogenized limit can be obtained for (1). Indeed, the homogenized problem is a parabolic fourth-order equation.

Finally, Section 9 is devoted to an extension of our previous results to a different model, namely we consider a fourth-order equation. We first obtain homogenized limits similar to those of Section 3 but with a fourth-order operator instead of a second-order one. Then, under a different assumption on the first Bloch eigenvalue, we prove that a second-order homogenized limit can also be obtained (a situation which is symmetric from that in Section 8). Our method could be generalized to other models. In particular, its application to the Schrödinger equation is of paramount interest. However, since much more can be deduced in the Schrödinger case, we address this problem in a separate work [9].

There are several motivations for studying the homogenization of the singularly perturbed system (1). First, (1) is a model of reaction-diffusion equations in periodic media (like a porous medium or a crystal in solid state 
physics) and the large potential is classical when studying long time asymptotics. Second, the spectral problem for (1) is an usual model in nuclear reactor physics, the so-called simplified transport equation. This is a set of diffusion equations for the even moments of the neutron flux (moments with respect to the angular velocity variable). One of the main features of this simplified transport system is that it does not satisfy a maximum principle. So our work is the first rigorous study of homogenization for this problem, which is of paramount interest for fast numerical computations in the nuclear industry (see [27] for more details and numerical applications). Third, as a limit case of large potentials we recover perforated domains with periodic holes supporting Dirichlet boundary conditions (take $c=+\infty$ in the holes and $c=0$ elsewhere). In such a case the term of order $\epsilon^{-2}$ disappear from the equation (1) although there is still a singular perturbation due to the presence of Dirichlet holes. The scalar setting, $K=1$, was studied in [28] and we extend this result to the vector-valued case. One possible application is the study of a composite material with fixed incusions in the context of linear elasticity. Fourth, even in the case when $c \equiv 0$ (i.e. without singular perturbation) our homogenization result for initial data concentrating at the bottom of high level Bloch bands is new and can be seen as a type of corrector result for capturing an initial layer in time in the context of classical homogenization [12] (see Remark 7.4).

\section{Notations and Bloch decomposition}

We first give our precise notations and assumptions on the coefficients $A(y)$ and $c(y)$ involved in equation (1). Our tensorial notations are the following. Recall that $N$ is the space dimension, and $K$ is the system dimension, i.e. all unknown functions are defined with values in $\mathbb{R}^{K}$. We adopt the convention that Latin indices $i, j$ belong to $\{1, . ., N\}$, i.e. refer to spatial coordinates, while Greek indices $\alpha, \beta$ vary in $\{1, . ., K\}$. The $K \times K$ matrices $c$ and $d$ are symmetric, with entries $c_{\alpha \beta}, d_{\alpha \beta}$ respectively, and have no specific positivity properties. The tensor $A$ acts on $K \times N$ matrices. Denoting by $\left(u_{\alpha}\right)_{1 \leq \alpha \leq K}$ the components of a vector-valued function $u$, its gradient is the $K \times N$ matrix $\nabla u$ defined by its entries

$$
\nabla u=\left(\frac{\partial u_{\alpha}}{\partial x_{i}}\right)_{1 \leq \alpha \leq K, 1 \leq i \leq N},
$$


and the product $A \nabla u$ is also a $K \times N$ matrix defined with the Einstein summation convention by

$$
A \nabla u=\left(A_{\alpha \beta i j} \frac{\partial u_{\alpha}}{\partial x_{i}}\right)_{1 \leq \beta \leq K, 1 \leq j \leq N} .
$$

The tensor $A$ is symmetric in the sense that

$$
A \xi \cdot \xi^{\prime}=A \xi^{\prime} \cdot \xi \text { for any } \xi, \xi^{\prime} \in \mathbb{R}^{K \times N},
$$

and it is uniformly coercive, i.e. there exists $\nu>0$ such that for a.e. $y \in \mathbb{T}^{N}$

$$
A(y) \xi \cdot \xi \geq \nu|\xi|^{2} \text { for any } \xi \in \mathbb{R}^{K \times N} .
$$

We assume that $A(y)$ and $c(y)$ are measurable bounded periodic functions, i.e. their entries belong to $L^{\infty}\left(\mathbb{T}^{N}\right)$, while $d(x, y)$ is measurable and bounded with respect to $x$, and periodic continuous with respect to $y$, i.e. its entries belong to $L^{\infty}\left(\Omega ; C\left(\mathbb{T}^{N}\right)\right)$ (other assumptions are possible).

A formal two-scale asymptotic expansion (in the spirit of [11]) shows that the leading term in the ansatz of $u_{\epsilon}$ is the solution of an equation in the unit cell $\mathbb{T}^{N}$. Therefore, we need to study a microscopic version of (1). It turns out that the key cell problem is the following Bloch (or shifted) spectral cell equation

$$
-\left(\operatorname{div}_{y}+2 i \pi \theta\right)\left(A(y)\left(\nabla_{y}+2 i \pi \theta\right) \psi_{n}\right)+c(y) \psi_{n}=\lambda_{n}(\theta) \psi_{n} \quad \text { in } \mathbb{T}^{N},
$$

which, as a compact self-adjoint complex-valued operator on $L^{2}\left(\mathbb{T}^{N}\right)$, admits a countable sequence of real increasing eigenvalues $\left(\lambda_{n}\right)_{n \geq 1}$ and normalized eigenfunctions $\left(\psi_{n}\right)_{n \geq 1}$ with $\left\|\psi_{n}\right\|_{L^{2}\left(\mathbb{T}^{N}\right)}=1$. The dual parameter $\theta$ is called the Bloch frequency and it runs in the dual cell of $\mathbb{T}^{N}$, i.e. by periodicity it is enough to consider $\theta \in \mathbb{T}^{N}$. We refer to [11, [17] for more details about the Bloch spectral problem (8).

Our main assumption is that there exists a Bloch parameter $\theta_{0} \in \mathbb{T}^{N}$ such that

$$
\begin{cases}(i) & \theta_{0} \text { is the unique minimizer of } \lambda_{1}(\theta) \text { in } \mathbb{T}^{N} \\ \text { (ii }) & \lambda_{1}\left(\theta_{0}\right) \text { is a simple eigenvalue, } \\ \text { (iii) } & \text { the Hessian matrix } \nabla_{\theta} \nabla_{\theta} \lambda_{1}\left(\theta_{0}\right) \text { is positive definite. }\end{cases}
$$


Remark 2.1 In the scalar case, $K=1$, assumption (9) is satisfied with $\theta_{0}=0$. Indeed, by using the maximum principle, it is easily seen that the minimum of $\lambda_{1}(\theta)$ is uniquely attained at 0 , and then that the Hessian matrix $\nabla_{\theta} \nabla_{\theta} \lambda_{1}(0)$, being equal to the usual homogenized matrix (see e.g. [18]), is positive definite. On the other hand, for any $K \geq 1$ and in the absence of zero-order term, i.e. $c=0$, it is easy to check that $\theta_{0}=0$ is the unique minimizer of $\lambda_{1}(\theta)$ (however, $\lambda_{1}(0)$ may be not simple and/or the Hessian matrix may be not positive definite). In full generality, there always exist a minimizer of $\lambda_{1}(\theta)$ but it may be non-unique and $\lambda_{1}\left(\theta_{0}\right)$ has no reason to be simple (although there are some results of generic simplicity in similar contexts, see [1]).

Remark 2.2 In a slightly different context, namely for a system of linear elasticity which is not uniformyl elliptic but simply satisfies the Hadamard ellipticity condition (in other words the associed energy is rank-one convex but not convex), there are numerical and physical evidences that the minimal value $\theta_{0}$ in (9) is not zero [19].

Remark 2.3 Assumption (9) can be slightly weakened, see Remarks 4.5 and 4.6.

Under assumption (9) it is a classical matter to prove that the first eigencouple of $(8)$ is analytic at $\theta_{0}$. Introducing the operator $\mathbb{A}(\theta)$ defined on $L^{2}\left(\mathbb{T}^{N}\right)^{K}$ by

$$
\mathbb{A}(\theta) \psi=-\left(\operatorname{div}_{y}+2 i \pi \theta\right)\left(A(y)\left(\nabla_{y}+2 i \pi \theta\right) \psi\right)+c(y) \psi-\lambda_{1}(\theta) \psi,
$$

it is easy to compute the derivatives of (8) for $n=1$. Denoting by $\left(e_{k}\right)_{1 \leq k \leq N}$ the canonical basis of $\mathbb{R}^{N}$, the first derivative satisfies

$\mathbb{A}(\theta) \frac{\partial \psi_{1}}{\partial \theta_{k}}=2 i \pi e_{k} A(y)\left(\nabla_{y}+2 i \pi \theta\right) \psi_{1}+\left(\operatorname{div}_{y}+2 i \pi \theta\right)\left(A(y) 2 i \pi e_{k} \psi_{1}\right)+\frac{\partial \lambda_{1}}{\partial \theta_{k}}(\theta) \psi_{1}$, 
and the second derivative is

$$
\begin{aligned}
\mathbb{A}(\theta) \frac{\partial^{2} \psi_{1}}{\partial \theta_{k} \partial \theta_{l}} & =2 i \pi e_{k} A(y)\left(\nabla_{y}+2 i \pi \theta\right) \frac{\partial \psi_{1}}{\partial \theta_{l}}+\left(\operatorname{div}_{y}+2 i \pi \theta\right)\left(A(y) 2 i \pi e_{k} \frac{\partial \psi_{1}}{\partial \theta_{l}}\right) \\
& +2 i \pi e_{l} A(y)\left(\nabla_{y}+2 i \pi \theta\right) \frac{\partial \psi_{1}}{\partial \theta_{k}}+\left(\operatorname{div}_{y}+2 i \pi \theta\right)\left(A(y) 2 i \pi e_{l} \frac{\partial \psi_{1}}{\partial \theta_{k}}\right) \\
& +\frac{\partial \lambda_{1}}{\partial \theta_{k}}(\theta) \frac{\partial \psi_{1}}{\partial \theta_{l}}+\frac{\partial \lambda_{1}}{\partial \theta_{l}}(\theta) \frac{\partial \psi_{1}}{\partial \theta_{k}} \\
& -4 \pi^{2} e_{k} A(y) e_{l} \psi_{1}-4 \pi^{2} e_{l} A(y) e_{k} \psi_{1}+\frac{\partial^{2} \lambda_{1}}{\partial \theta_{l} \partial \theta_{k}}(\theta) \psi_{1}
\end{aligned}
$$

For $\theta=\theta_{0}$ we have $\nabla_{\theta} \lambda_{1}\left(\theta_{0}\right)=0$, thus equations (11) and (12) simplify and we find

$$
\frac{\partial \psi_{1}}{\partial \theta_{k}}=2 i \pi \zeta_{k}, \quad \frac{\partial^{2} \psi_{1}}{\partial \theta_{k} \partial \theta_{l}}=-4 \pi^{2} \chi_{k l},
$$

where $\zeta_{k}$ is the solution of

$$
\mathbb{A}\left(\theta_{0}\right) \zeta_{k}=e_{k} A(y)\left(\nabla_{y}+2 i \pi \theta_{0}\right) \psi_{1}+\left(\operatorname{div}_{y}+2 i \pi \theta_{0}\right)\left(A(y) e_{k} \psi_{1}\right) \quad \text { in } \mathbb{T}^{N},
$$

and $\chi_{k l}$ is the solution of

$$
\begin{aligned}
\mathbb{A}\left(\theta_{0}\right) \chi_{k l}= & e_{k} A(y)\left(\nabla_{y}+2 i \pi \theta_{0}\right) \zeta_{l}+\left(\operatorname{div}_{y}+2 i \pi \theta_{0}\right)\left(A(y) e_{k} \zeta_{l}\right) \\
& +e_{l} A(y)\left(\nabla_{y}+2 i \pi \theta_{0}\right) \zeta_{k}+\left(\operatorname{div}_{y}+2 i \pi \theta_{0}\right)\left(A(y) e_{l} \zeta_{k}\right) \\
& +e_{k} A(y) e_{l} \psi_{1}+e_{l} A(y) e_{k} \psi_{1}-\frac{1}{4 \pi^{2}} \frac{\partial^{2} \lambda_{1}}{\partial \theta_{l} \partial \theta_{k}}\left(\theta_{0}\right) \psi_{1} \quad \text { in } \mathbb{T}^{N} .
\end{aligned}
$$

There exists a unique solution of (14), up to the addition of a multiple of $\psi_{1}$. Indeed, the right hand side of (14) satisfies the required compatibility condition (i.e. it is orthogonal to $\psi_{1}$ ) because $\zeta_{k}$ is just a multiple of the partial derivative of $\psi_{1}$ with respect to $\theta_{k}$ which necessarily exists, see (11). On the same token, there exists a unique solution of (15), up to the addition of a multiple of $\psi_{1}$. The compatibility condition of (15) yields a formula for the Hessian matrix $\nabla_{\theta} \nabla_{\theta} \lambda_{1}\left(\theta_{0}\right)$.

We now recall some results on the Bloch decomposition associated to the spectral problem (8) (see e.g. [11, [17]). 
Lemma 2.4 Let $u(y) \in L^{2}\left(\mathbb{R}^{N}\right)^{K}$. Define $\alpha_{k}(\theta)=\int_{\mathbb{T}^{N}} u(y) \cdot \bar{\psi}_{k}(y, \theta) e^{-2 i \pi \theta \cdot y} d y$. Then,

$$
u(y)=\sum_{k \geq 1} \int_{\mathbb{T}^{N}} \alpha_{k}(\theta) \psi_{k}(y, \theta) e^{2 i \pi \theta \cdot y} d \theta .
$$

Furthermore, if $v(y)=\sum_{k \geq 1} \int_{\mathbb{T}^{N}} \beta_{k}(\theta) \psi_{k}(y, \theta) e^{2 i \pi \theta \cdot y} d \theta$ in $L^{2}\left(\mathbb{R}^{N}\right)^{K}$, we have

$$
\int_{\mathbb{R}^{N}} u(y) \cdot \bar{v}(y) d y=\sum_{k \geq 1} \int_{\mathbb{T}^{N}} \alpha_{k}(\theta) \bar{\beta}_{k}(\theta) d \theta .
$$

In the sequel we shall need a rescaled version of Lemma 2.4 that we now describe. Upon the change of variable $y=\frac{x}{\epsilon}$, we define $u^{\epsilon}(x)=\epsilon^{-N / 2} u(y)$ which satisfies $\left\|u^{\epsilon}\right\|_{L^{2}\left(\mathbb{R}^{N}\right)^{K}}=\|u\|_{L^{2}\left(\mathbb{R}^{N}\right)^{K}}$. Applying Lemma 2.4 we deduce the following rescaled Bloch transform

$$
u_{\epsilon}(x)=\sum_{k \geq 1} \int_{\epsilon^{-1} \mathbb{T}^{N}} \alpha_{k}^{\epsilon}(\eta) \psi_{k}\left(\frac{x}{\epsilon}, \theta_{0}+\epsilon \eta\right) e^{2 i \pi \eta \cdot x} e^{2 i \pi \frac{\theta_{0} \cdot x}{\epsilon}} d \eta
$$

with $\eta=\frac{\theta-\theta_{0}}{\epsilon}$ and $\alpha_{k}^{\epsilon}(\eta)=\epsilon^{N / 2} \alpha_{k}(\theta)$. The same orthogonality property holds true

$$
\int_{\mathbb{R}^{N}} u^{\epsilon}(x) \cdot \bar{v}^{\epsilon}(x) d x=\sum_{k \geq 1} \int_{\epsilon^{-1} \mathbb{T}^{N}} \alpha_{k}^{\epsilon}(\eta) \bar{\beta}_{k}^{\epsilon}(\eta) d \eta
$$

\section{Main results}

Let $\Omega \subset \mathbb{R}^{N}$ be an open set (bounded or not). Let $0<T<+\infty$ be a final time. We first consider the following parabolic problem

$$
\begin{cases}\frac{\partial u_{\epsilon}}{\partial t}-\operatorname{div}\left(A\left(\frac{x}{\epsilon}\right) \nabla u_{\epsilon}\right)+\left(\frac{c\left(\frac{x}{\epsilon}\right)}{\epsilon^{2}}+d\left(x, \frac{x}{\epsilon}\right)\right) u_{\epsilon}=0 & \text { in } \Omega \times(0, T), \\ u_{\epsilon}=0 & \text { on } \partial \Omega \times(0, T), \\ u_{\epsilon}(t=0, x)=u_{\epsilon}^{0}(x) & \text { in } \Omega .\end{cases}
$$

The unknown $u_{\epsilon}(t, x)$ is vector-valued, i.e. it is a function from $(0, T) \times$ $\Omega$ into $\mathbb{R}^{K}$ with $K \geq 1$. Assuming that the initial data $u_{\epsilon}^{0}$ belongs to $L^{2}(\Omega)^{K}$ it is a classical result that there exists a unique solution of 17 in $C\left((0, T) ; L^{2}(\Omega)^{K}\right) \cap L^{2}\left((0, T) ; H_{0}^{1}(\Omega)^{K}\right)$. 
Since the matrix $c$ does not satisfy any positivity property, we can not obtain any a priori estimate directly from (17). On the other hand, the cell spectral problem and assumption (9) indicate that $\lambda_{1}\left(\theta_{0}\right)$ governs the time decay (or growth, according to its sign) of the solution $u_{\epsilon}$. Therefore, we first perform a time renormalization in the spirit of the factorization principle (3) and we introduce a new unknown

$$
\tilde{u}_{\epsilon}(t, x)=e^{\frac{\lambda_{1}\left(\theta_{0}\right) t}{\epsilon^{2}}} u_{\epsilon}(t, x)
$$

which satisfies

$$
\begin{cases}\frac{\partial \tilde{u}_{\epsilon}}{\partial t}-\operatorname{div}\left(A\left(\frac{x}{\epsilon}\right) \nabla \tilde{u}_{\epsilon}\right)+\frac{c\left(\frac{x}{\epsilon}\right)-\lambda_{1}\left(\theta_{0}\right)}{\epsilon^{2}} \tilde{u}_{\epsilon}+d\left(x, \frac{x}{\epsilon}\right) \tilde{u}_{\epsilon}=0 & \text { in } \Omega \times(0, T), \\ \tilde{u}_{\epsilon}=0 & \text { on } \partial \Omega \times(0, T), \\ \tilde{u}_{\epsilon}(t=0, x)=u_{\epsilon}^{0}(x) & \text { in } \Omega .\end{cases}
$$

Then, we can obtain the following a priori estimate.

Lemma 3.1 There exists a constant $C>0$ which does not depend on $\epsilon$ (but may depend on $T$ ) such that the solution of (19) satisfies

$$
\left\|\tilde{u}_{\epsilon}\right\|_{L^{\infty}\left((0, T) ; L^{2}(\Omega)^{K}\right)}+\epsilon\left\|\nabla \tilde{u}_{\epsilon}\right\|_{L^{2}((0, T) \times \Omega)^{N \times K}} \leq C\left\|u_{\epsilon}^{0}\right\|_{L^{2}(\Omega)^{K}} .
$$

Theorem 3.2 Assume (9) and that the initial data $u_{\epsilon}^{0} \in L^{2}(\Omega)^{K}$ is of the form

$$
u_{\epsilon}^{0}(x)=\psi_{1}\left(\frac{x}{\epsilon}, \theta_{0}\right) e^{2 i \pi \frac{\theta_{0} \cdot x}{\epsilon}} v^{0}(x),
$$

with $v^{0} \in L^{2}(\Omega)$. The solution of (17) can be written as

$$
u_{\epsilon}(t, x)=e^{-\frac{\lambda_{1}\left(\theta_{0}\right) t}{\epsilon^{2}}}\left(\psi_{1}\left(\frac{x}{\epsilon}, \theta_{0}\right) e^{2 i \pi \frac{\theta_{0} \cdot x}{\epsilon}} v_{\epsilon}(t, x)+r_{\epsilon}(t, x)\right),
$$

where $r_{\epsilon}$ is a vector-valued remainder term such that

$$
\lim _{\epsilon \rightarrow 0}\left\|r_{\epsilon}\right\|_{L^{2}((0, T) \times \omega)^{K}}=0 \text { for any compact set } \omega \subset \mathbb{R}^{N},
$$

and $v_{\epsilon}$ is a scalar sequence which converges weakly in $L^{2}\left((0, T) ; H^{1}(\Omega)\right)$ to the solution $v$ of the scalar homogenized problem

$$
\begin{cases}\frac{\partial v}{\partial t}-\operatorname{div}\left(A^{*} \nabla v\right)+d^{*}(x) v=0 & \text { in } \Omega \times(0, T), \\ v=0 & \text { on } \partial \Omega \times(0, T), \\ v(t=0, x)=v^{0}(x) & \text { in } \Omega,\end{cases}
$$

with $A^{*}=\frac{1}{4 \pi^{2}} \nabla_{\theta} \nabla_{\theta} \lambda_{1}\left(\theta_{0}\right)$ and $d^{*}(x)=\int_{\mathbb{T}^{N}} d(x, y) \psi_{1}(y) \cdot \bar{\psi}_{1}(y) d y$. 
Remark 3.3 Of course, if $\Omega$ is bounded, one can take $\omega=\Omega$ in (23).

Remark 3.4 Assumption (21) is not necessary for proving Theorem 3.2. For example, it still holds true with the weaker assumption that $u_{\epsilon}^{0}(x) e^{-2 i \pi \frac{\theta_{0} \cdot x}{\epsilon}}$ two-scale converges to $\psi_{1}\left(y, \theta_{0}\right) v^{0}(x)$ with $v^{0} \in L^{2}(\Omega)$ (see [2], [23] for the notion of two-scale convergence). Furthermore, for any kind of initial data we can still obtain a similar result, but the homogenized initial condition $v^{0}$ is just defined as some type of weak two-scale limit (which may well be zero). In other words, there is no need to have "well-prepared" initial data in Theorem 3.2.

Remark 3.5 Theorem 3.2 still holds true if we add to equation (17) a nonlinear term of order $\epsilon^{0}$. Typically, we can add a non-linear term of the type $g\left(x, \frac{x}{\epsilon}, u_{\epsilon}\right)$ where $g(x, y, \xi)$ is an homogeneous of degree one, Lipschitz function with respect to $\xi$ such that

$$
\left|g(x, y, \xi)-g\left(x, y, \xi^{\prime}\right)\right| \leq C\left|\xi-\xi^{\prime}\right|, \quad g(x, y, t \xi)=\operatorname{tg}(x, y, \xi) \quad \forall t>0 .
$$

In such a case, the homogenized problem (24) has an additional zero-order term which is $g^{*}(x, v)$ with $g^{*}(x, v)=\int_{\mathbb{T}^{N}} g\left(x, y, \psi_{1}\left(y, \theta_{0}\right) v\right) \cdot \bar{\psi}_{1}\left(y, \theta_{0}\right) d y$. Similarly, it is possible to add to (17) a source term of the type

$$
f_{\epsilon}(t, x)=e^{-\frac{\lambda_{1}\left(\theta_{0}\right) t}{\epsilon^{2}}} e^{2 i \pi \frac{\theta_{0} \cdot x}{\epsilon}} f\left(t, x, \frac{x}{\epsilon}\right) .
$$

It yields a source term $f^{*}(t, x)=\int_{\mathbb{T}^{N}} f(t, x, y) \cdot \bar{\psi}_{1}(y) d y$ in the homogenized equation (24).

We now consider the eigenvalue problem in a bounded domain $\Omega$ (the case of $\Omega=\mathbb{R}^{N}$ is also discussed in Section 5

$$
\begin{cases}-\operatorname{div}\left(A\left(\frac{x}{\epsilon}\right) \nabla u_{\epsilon}\right)+\left(\frac{c\left(\frac{x}{\epsilon}\right)}{\epsilon^{2}}+d\left(x, \frac{x}{\epsilon}\right)\right) u_{\epsilon}=\lambda_{\epsilon} u_{\epsilon} & \text { in } \Omega, \\ u_{\epsilon}=0 & \text { on } \partial \Omega .\end{cases}
$$

Since $\Omega$ is assumed to be bounded, problem 25 has a discrete spectrum

$$
\lambda_{1}^{\epsilon} \leq \lambda_{2}^{\epsilon} \leq \ldots \leq \lambda_{n}^{\epsilon} \ldots \rightarrow+\infty,
$$

with eigenfunctions denoted by $u_{k}^{\epsilon}$, normalized by $\left\|u_{k}^{\epsilon}\right\|_{L^{2}(\Omega)^{K}}=1$. 
Theorem 3.6 For each $k \geq 1$ we have

$$
\lambda_{k}^{\epsilon}=\frac{\lambda_{1}\left(\theta_{0}\right)}{\epsilon^{2}}+\mu_{k}+o(1) \quad \text { with } \lim _{\epsilon \rightarrow 0} o(1)=0,
$$

and the corresponding eigenvector $u_{k}^{\epsilon}(x)$ admits the representation

$$
u_{k}^{\epsilon}(x)=\psi_{1}\left(\frac{x}{\epsilon}, \theta_{0}\right) e^{2 i \pi \frac{\theta_{0} \cdot x}{\epsilon}} v_{k}^{\epsilon}(x)+r_{k}^{\epsilon}(x)
$$

where

$$
\lim _{\epsilon \rightarrow 0}\left\|r_{k}^{\epsilon}\right\|_{L^{2}(\Omega)^{K}}=0, \quad\left\|v_{k}^{\epsilon}\right\|_{H^{1}(\Omega)} \leq C, \quad \lim _{\epsilon \rightarrow 0}\left\|v_{k}^{\epsilon}\right\|_{L^{2}(\Omega)}=1,
$$

and any limit point $v_{k}$, as $\epsilon \rightarrow 0$, of the scalar sequence $v_{k}^{\epsilon}$ is a normalized eigenfunction associated to the $k$-th eigenvalue $\mu_{k}$ of the scalar homogenized spectral problem

$$
\begin{cases}-\operatorname{div}\left(A^{*} \nabla v\right)+d^{*}(x) v=\mu v & \text { in } \Omega, \\ v=0 & \text { on } \partial \Omega,\end{cases}
$$

with $A^{*}=\frac{1}{4 \pi^{2}} \nabla_{\theta} \nabla_{\theta} \lambda_{1}\left(\theta_{0}\right)$ and $d^{*}(x)=\int_{\mathbb{T}^{N}} d(x, y) \psi_{1}(y) \cdot \bar{\psi}_{1}(y) d y$.

Furthermore, if $\mu_{k}$ is a simple eigenvalue of (27), the entire sequence $v_{k}^{\epsilon}$ converges to the homogenized eigenfunction $v_{k}$.

Finally we address the following hyperbolic problem

$$
\begin{cases}\frac{\partial^{2} u_{\epsilon}}{\partial t^{2}}-\operatorname{div}\left(A\left(\frac{x}{\epsilon}\right) \nabla u_{\epsilon}\right)+\frac{c\left(\frac{x}{\epsilon}\right)}{\epsilon^{2}} u_{\epsilon}=0 & \text { in } \Omega \times(0, T), \\ u_{\epsilon}=0 & \text { on } \partial \Omega \times(0, T), \\ u_{\epsilon}(t=0, x)=u_{\epsilon}^{0}(x) & \text { in } \Omega, \\ \frac{\partial u_{\epsilon}}{\partial t}(t=0, x)=u_{\epsilon}^{1}(x) & \text { in } \Omega .\end{cases}
$$

The unknown $u_{\epsilon}(t, x)$ is vector-valued, i.e. it is a function from $(0, T) \times \Omega$ into $\mathbb{R}^{K}$ with $K \geq 1$. Assuming that the initial data are $u_{\epsilon}^{0} \in H_{0}^{1}(\Omega)^{K}$ and $\left.u_{\epsilon}^{1} \in L^{2}(\Omega)^{K}, 28\right)$ admits a unique solution $u_{\epsilon} \in C\left([0, T] ; H_{0}^{1}(\Omega)^{K}\right) \cap$ $C^{1}\left([0, T] ; L^{2}(\Omega)^{K}\right)$. The scalar case $K=1$ was addressed in [3]. Depending on the sign of the minimal eigenvalue $\lambda_{1}\left(\theta_{0}\right)$ of the cell problem (8), we obtain different asymptotic behavior for $(28)$. We begin with the case $\lambda_{1}\left(\theta_{0}\right)=0$ which does not require any time renormalization. 
Theorem 3.7 Assume (9), $\lambda_{1}\left(\theta_{0}\right)=0$ and that the initial data are of the form

$$
\begin{aligned}
& u_{\epsilon}^{0}(x)=\psi_{1}\left(\frac{x}{\epsilon}, \theta_{0}\right) e^{2 i \pi \frac{\theta_{0} \cdot x}{\epsilon}} v^{0}(x) \in H_{0}^{1}(\Omega)^{K}, \\
& u_{\epsilon}^{1}(x)=\psi_{1}\left(\frac{x}{\epsilon}, \theta_{0}\right) e^{2 i \pi \frac{\theta_{0} \cdot x}{\epsilon}} v^{1}(x) \in L^{2}(\Omega)^{K}
\end{aligned}
$$

with $v^{0} \in H_{0}^{1}(\Omega)$ and $v^{1} \in L^{2}(\Omega)$. The solution of (28) can be written as

$$
u_{\epsilon}(t, x)=\psi_{1}\left(\frac{x}{\epsilon}, \theta_{0}\right) e^{2 i \pi \frac{\theta_{0} \cdot x}{\epsilon}} v_{\epsilon}(t, x)+r_{\epsilon}(t, x),
$$

where $r_{\epsilon}$ is a vector-valued remainder term such that

$$
\lim _{\epsilon \rightarrow 0}\left\|r_{\epsilon}\right\|_{L^{2}((0, T) \times \omega)^{K}}=0 \text { for any compact set } \omega \subset \mathbb{R}^{N}
$$

and $v_{\epsilon}$ is a scalar sequence which converges weakly in $L^{2}\left((0, T) ; H^{1}(\Omega)\right)$ to the solution $v$ of the scalar homogenized problem

$$
\begin{cases}\frac{\partial^{2} v}{\partial t^{2}}-\operatorname{div}\left(A^{*} \nabla v\right)=0 & \text { in } \Omega \times(0, T) \\ v=0 & \text { on } \partial \Omega \times(0, T) \\ v(t=0, x)=v^{0}(x) & \text { in } \Omega \\ \frac{\partial v}{\partial t}(t=0, x)=v^{1}(x) & \text { in } \Omega\end{cases}
$$

with $A^{*}=\frac{1}{4 \pi^{2}} \nabla_{\theta} \nabla_{\theta} \lambda_{1}\left(\theta_{0}\right)$.

When $\lambda_{1}\left(\theta_{0}\right) \neq 0$, we can not homogenize directly (28). As in the scalar case [3] we must rather perform a time rescaling and consider large times of order $\epsilon^{-1}$. In other words, instead of (28) we now consider

$$
\begin{cases}\epsilon^{2} \frac{\partial^{2} u_{\epsilon}}{\partial t^{2}}-\operatorname{div}\left(A\left(\frac{x}{\epsilon}\right) \nabla u_{\epsilon}\right)+\frac{c\left(\frac{x}{\epsilon}\right)}{\epsilon^{2}} u_{\epsilon}=0 & \text { in } \Omega \times(0, T) \\ u_{\epsilon}=0 & \text { on } \partial \Omega \times(0, T) \\ u_{\epsilon}(t=0, x)=u_{\epsilon}^{0}(x) & \text { in } \Omega \\ \frac{\partial u_{\epsilon}}{\partial t}(t=0, x)=u_{\epsilon}^{1}(x) & \text { in } \Omega .\end{cases}
$$

Let us first assume that $\lambda_{1}\left(\theta_{0}\right)<0$. We perform a time renormalization analogous to (18) and we introduce a new unknown

$$
\tilde{u}_{\epsilon}(t, x)=e^{-\frac{\sqrt{-\lambda_{1}\left(\theta_{0}\right) t}}{\epsilon^{2}}} u_{\epsilon}(t, x)
$$


which satisfies

$$
\begin{cases}\epsilon^{2} \frac{\partial^{2} \tilde{u}_{\epsilon}}{\partial t^{2}}+2 \sqrt{-\lambda_{1}\left(\theta_{0}\right)} \frac{\partial \tilde{u}_{\epsilon}}{\partial t}-\operatorname{div}\left(A\left(\frac{x}{\epsilon}\right) \nabla \tilde{u}_{\epsilon}\right)+\frac{c\left(\frac{x}{\epsilon}\right)-\lambda_{1}\left(\theta_{0}\right)}{\epsilon^{2}} \tilde{u}_{\epsilon}=0 & \text { in } \Omega \times(0, T), \\ \tilde{u}_{\epsilon}=0 & \text { on } \partial \Omega \times(0, T), \\ \tilde{u}_{\epsilon}(t=0, x)=u_{\epsilon}^{0}(x) & \text { in } \Omega, \\ \frac{\partial \tilde{u}_{\epsilon}}{\partial t}(t=0, x)=u_{\epsilon}^{1}(x)-\frac{\sqrt{-\lambda_{1}\left(\theta_{0}\right)}}{\epsilon^{2}} u_{\epsilon}^{0}(x) & \text { in } \Omega .\end{cases}
$$

In this case we obtain a parabolic homogenized equation.

Theorem 3.8 Assume (9), $\lambda_{1}\left(\theta_{0}\right)<0$ and that the initial data is

$$
u_{\epsilon}^{0}(x)=\psi_{1}\left(\frac{x}{\epsilon}, \theta_{0}\right) e^{2 i \pi \frac{\theta_{0} \cdot x}{\epsilon}} v^{0}(x) \in H_{0}^{1}(\Omega)^{K},
$$

with $v^{0} \in H_{0}^{1}(\Omega)$, and that $\epsilon^{2} u_{\epsilon}^{1}(x)$ is bounded in $L^{2}(\Omega)^{K}$ while $\epsilon^{2} \psi_{1}\left(\frac{x}{\epsilon}, \theta_{0}\right)$. $u_{\epsilon}^{1}(x)$ converges weakly to 0 in $L^{2}(\Omega)$. The solution of (35) can be written as

$$
u_{\epsilon}(t, x)=e^{\frac{\sqrt{-\lambda_{1}\left(\theta_{0}\right) t}}{\epsilon^{2}}}\left(\psi_{1}\left(\frac{x}{\epsilon}, \theta_{0}\right) e^{2 i \pi \frac{\theta_{0} \cdot x}{\epsilon}} v_{\epsilon}(t, x)+r_{\epsilon}(t, x)\right)
$$

where $r_{\epsilon}$ is a vector-valued remainder term such that

$$
\lim _{\epsilon \rightarrow 0}\left\|r_{\epsilon}\right\|_{L^{2}((0, T) \times \omega)^{K}}=0 \text { for any compact set } \omega \subset \mathbb{R}^{N},
$$

and $v_{\epsilon}$ converges weakly in $L^{2}\left((0, T) ; H^{1}(\Omega)\right)$ to the solution $v$ of the scalar homogenized problem

$$
\begin{cases}2 \sqrt{-\lambda_{1}\left(\theta_{0}\right)} \frac{\partial v}{\partial t}-\operatorname{div}\left(A^{*} \nabla v\right)=0 & \text { in } \Omega \times(0, T), \\ v=0 & \text { on } \partial \Omega \times(0, T), \\ v(t=0, x)=\frac{1}{2} v^{0}(x) & \text { in } \Omega\end{cases}
$$

with $A^{*}=\frac{1}{2} \nabla_{\theta} \nabla_{\theta} \lambda_{1}\left(\theta_{0}\right)$.

Remark 3.9 The one half factor in front of the initial data in the homogenized problem (39) is quite surprising. It arises because the initial velocity in (35) contains some contribution of $u_{\epsilon}^{0}$. As already explained in the scalar case [3], there is an initial layer in time in (35) which is not taken into account by Theorem 3.8 . 
Let us now assume that $\lambda_{1}\left(\theta_{0}\right)>0$. We perform another time renormalization and we introduce a new unknown

$$
\tilde{u}_{\epsilon}(t, x)=e^{-i \frac{\sqrt{\lambda_{1}\left(\theta_{0}\right)} t}{\epsilon^{2}}} u_{\epsilon}(t, x)
$$

which satisfies

$$
\begin{cases}\epsilon^{2} \frac{\partial^{2} \tilde{u}_{\epsilon}}{\partial t^{2}}+2 i \sqrt{\lambda_{1}\left(\theta_{0}\right)} \frac{\partial \tilde{u}_{\epsilon}}{\partial t}-\operatorname{div}\left(A\left(\frac{x}{\epsilon}\right) \nabla \tilde{u}_{\epsilon}\right)+\frac{c\left(\frac{x}{\epsilon}\right)-\lambda_{1}\left(\theta_{0}\right)}{\epsilon^{2}} \tilde{u}_{\epsilon}=0 & \text { in } \Omega \times(0, T), \\ \tilde{u}_{\epsilon}=0 & \text { on } \partial \Omega \times(0, T), \\ \tilde{u}_{\epsilon}(t=0, x)=u_{\epsilon}^{0}(x) & \text { in } \Omega, \\ \frac{\partial \tilde{u}_{\epsilon}}{\partial t}(t=0, x)=u_{\epsilon}^{1}(x)-i \frac{\sqrt{\lambda_{1}\left(\theta_{0}\right)}}{\epsilon^{2}} u_{\epsilon}^{0}(x) & \text { in } \Omega .\end{cases}
$$

In this case we obtain a Schrödinger type homogenized equation.

Theorem 3.10 Assume (9), $\lambda_{1}\left(\theta_{0}\right)>0$ and that the initial data is

$$
u_{\epsilon}^{0}(x)=\psi_{1}\left(\frac{x}{\epsilon}, \theta_{0}\right) e^{2 i \pi \frac{\theta_{0} \cdot x}{\epsilon}} v^{0}(x) \in H_{0}^{1}(\Omega)^{K},
$$

with $v^{0} \in L^{2}(\Omega)$, and that $\epsilon^{2} u_{\epsilon}^{1}(x)$ is bounded in $L^{2}(\Omega)^{K}$ while $\epsilon^{2} \psi_{1}\left(\frac{x}{\epsilon}, \theta_{0}\right)$. $u_{\epsilon}^{1}(x)$ converges weakly to 0 in $L^{2}(\Omega)$. The solution of (35) can be written as

$$
u_{\epsilon}(t, x)=e^{i \frac{\sqrt{\lambda_{1}\left(\theta_{0}\right)} t}{\epsilon^{2}}} e^{2 i \pi \frac{\theta_{0} \cdot x}{\epsilon}} v_{\epsilon}(t, x),
$$

where $v_{\epsilon}$ two-scale converges to $\psi_{1}\left(y, \theta_{0}\right) v(t, x)$ and $v \in L^{2}\left((0, T) ; H_{0}^{1}(\Omega)\right)$ is the solution of the scalar homogenized problem

$$
\begin{cases}2 i \sqrt{\lambda_{1}\left(\theta_{0}\right)} \frac{\partial v}{\partial t}-\operatorname{div}\left(A^{*} \nabla v\right)=0 & \text { in } \Omega \times(0, T), \\ v=0 & \text { on } \partial \Omega \times(0, T), \\ v(t=0, x)=\frac{1}{2} v^{0}(x) & \text { in } \Omega,\end{cases}
$$

with $A^{*}=\frac{1}{2} \nabla_{\theta} \nabla_{\theta} \lambda_{1}\left(\theta_{0}\right)$.

Remark 3.11 All the results in the hyperbolic case (Theorems 3. 7, 3.8, and $3.10)$ hold true when we add a zero-order term of the type $d\left(x, \frac{x}{\epsilon}\right) u_{\epsilon}$, where $d(x, y)$ is a symmetric non-negative matrix with entries in $L^{\infty}\left(\Omega ; C\left(\mathbb{T}^{N}\right)\right)$. This yields a zero-order term in the homogenized problem which is precisely $d^{*}(x)=\int_{\mathbb{T}^{N}} d(x, y) \psi_{1}(y) \cdot \bar{\psi}_{1}(y) d y$. 


\section{Proofs in the parabolic case}

Notation: for any function $\phi(x, y)$ defined on $\mathbb{R}^{N} \times \mathbb{T}^{N}$, we denote by $\phi^{\epsilon}$ the function $\phi\left(x, \frac{x}{\epsilon}\right)$.

Proof of Lemma 3.1. We multiply equation (19) by $\tilde{u}_{\epsilon}$ and we integrate by parts to obtain

$$
\begin{aligned}
& \frac{1}{2} \int_{\Omega}\left|\tilde{u}_{\epsilon}(t, x)\right|^{2} d x-\frac{1}{2} \int_{\Omega}\left|u_{\epsilon}^{0}(x)\right|^{2} d x+\int_{0}^{t} \int_{\Omega} d\left(x \frac{x}{\epsilon}\right) \tilde{u}_{\epsilon} \cdot \tilde{u}_{\epsilon} d s d x \\
& +\int_{0}^{t} \int_{\Omega}\left(A\left(\frac{x}{\epsilon}\right) \nabla \tilde{u}_{\epsilon} \cdot \nabla \tilde{u}_{\epsilon}+\frac{c\left(\frac{x}{\epsilon}\right)-\lambda_{1}\left(\theta_{0}\right)}{\epsilon^{2}} \tilde{u}_{\epsilon} \cdot \tilde{u}_{\epsilon}\right) d s d x=0 .
\end{aligned}
$$

If we can check that the last integral in (45) is non negative, the lemma is proved by a standard Gronwall inequality. Extending $\tilde{u}_{\epsilon}$ by zero outside $\Omega$ and changing the variable as $y=\frac{x}{\epsilon}$, a sufficient condition is to prove that, for any $u \in H^{1}\left(\mathbb{R}^{N}\right)^{K}$,

$$
\int_{\mathbb{R}^{N}}\left(A(y) \nabla u \cdot \nabla u+\left(c(y)-\lambda_{1}\left(\theta_{0}\right)\right) u \cdot u\right) d y \geq 0 .
$$

Applying the Bloch decomposition of Lemma 2.4 to $u$ yields

$\int_{\mathbb{R}^{N}}\left(A(y) \nabla u \cdot \nabla u+\left(c(y)-\lambda_{1}\left(\theta_{0}\right)\right) u \cdot u\right) d y=\sum_{k \geq 1} \int_{\mathbb{T}^{N}}\left|\alpha_{k}(\theta)\right|^{2}\left(\lambda_{k}(\theta)-\lambda_{1}\left(\theta_{0}\right)\right) d \theta$

which is non negative by assumption (9).

Proof of Theorem 3.2. To simplify the exposition we forget the notation * for the solution $\tilde{u}_{\epsilon}$ of $(19)$. Equivalently, we could have subtracted from $c(y)$ an adequate constant, so that $\lambda_{1}\left(\theta_{0}\right)=0$ and $u_{\epsilon}=\tilde{u}_{\epsilon}$. Define a sequence $w_{\epsilon}$ by

$$
w_{\epsilon}(t, x)=u_{\epsilon}(t, x) e^{-2 i \pi \frac{\theta_{0} \cdot x}{\epsilon}} .
$$

By the a priori estimate of Lemma 3.1 we have

$$
\left\|w_{\epsilon}\right\|_{L^{\infty}\left((0, T) ; L^{2}(\Omega)^{K}\right)}+\epsilon\left\|\nabla w_{\epsilon}\right\|_{L^{2}((0, T) \times \Omega)^{K}} \leq C,
$$

and applying the compactness of two-scale convergence (see [2], [23]), up to a subsequence there exists a limit $w(t, x, y) \in L^{2}\left((0, T) \times \Omega ; H^{1}\left(\mathbb{T}^{N}\right)^{K}\right)$ such that

$$
w_{\epsilon} \stackrel{2 \mathrm{~s}}{\rightarrow} w \text { and } \epsilon \nabla w_{\epsilon} \stackrel{2 \mathrm{~s}}{\rightarrow} \nabla_{y} w
$$


in the sense of two-scale convergence.

First step. We multiply 190 by the complex conjugate of $\epsilon^{2} \phi\left(t, x, \frac{x}{\epsilon}\right) e^{2 i \pi \frac{\theta_{0} \cdot x}{\epsilon}}$ where $\phi(t, x, y)$ is a smooth test function defined on $[0, T) \times \Omega \times \mathbb{T}^{N}$, with compact support in $[0, T) \times \Omega$, and with values in $\mathbb{C}^{K}$. Integrating by parts this yields

$$
\begin{aligned}
& \epsilon^{2} \int_{\Omega} u_{\epsilon}^{0} \cdot \bar{\phi}^{\epsilon} e^{-2 i \pi \frac{\theta_{0} \cdot x}{\epsilon}} d x-\epsilon^{2} \int_{0}^{T} \int_{\Omega} w_{\epsilon} \cdot \frac{\partial \bar{\phi}^{\epsilon}}{\partial t} d t d x \\
& +\int_{0}^{T} \int_{\Omega} A^{\epsilon}\left(\epsilon \nabla+2 i \pi \theta_{0}\right) w_{\epsilon} \cdot\left(\epsilon \nabla-2 i \pi \theta_{0}\right) \bar{\phi}^{\epsilon} d t d x \\
& +\int_{0}^{T} \int_{\Omega}\left(c^{\epsilon}-\lambda_{1}\left(\theta_{0}\right)+\epsilon^{2} d^{\epsilon}\right) w_{\epsilon} \cdot \bar{\phi}^{\epsilon} d t d x=0 .
\end{aligned}
$$

Passing to the two-scale limit yields the variational formulation of

$$
-\left(\operatorname{div}_{y}+2 i \pi \theta\right)\left(A(y)\left(\nabla_{y}+2 i \pi \theta\right) w\right)+c(y) w=\lambda_{1}\left(\theta_{0}\right) w \quad \text { in } \mathbb{T}^{N} .
$$

By the simplicity of $\lambda_{1}\left(\theta_{0}\right)$, this implies that there exists a scalar function $v(t, x) \in L^{2}((0, T) \times \Omega)$ (possibly complex-valued) such that

$$
w(t, x, y)=v(t, x) \psi_{1}\left(y, \theta_{0}\right) .
$$

Second step. We multiply (19) by the complex conjugate of

$$
\Psi_{\epsilon}=e^{2 i \pi \frac{\theta_{0} \cdot x}{\epsilon}}\left(\psi_{1}\left(\frac{x}{\epsilon}, \theta_{0}\right) \phi(t, x)+\epsilon \sum_{k=1}^{N} \frac{\partial \phi}{\partial x_{k}}(t, x) \zeta_{k}\left(\frac{x}{\epsilon}\right)\right)
$$

where $\phi(t, x)$ is a smooth, compactly supported, test function defined from $[0, T) \times \Omega$ into $\mathbb{R}$, and $\zeta_{k}(y)$ is the solution of (14). After some algebra we 
found that

$$
\begin{aligned}
\int_{\Omega} A^{\epsilon} \nabla u_{\epsilon} \cdot \nabla \bar{\Psi}_{\epsilon} d x & =\int_{\Omega} A^{\epsilon}\left(\nabla+2 i \pi \frac{\theta_{0}}{\epsilon}\right)\left(\bar{\phi} w_{\epsilon}\right) \cdot\left(\nabla-2 i \pi \frac{\theta_{0}}{\epsilon}\right) \bar{\psi}_{1}^{\epsilon} \\
& +\epsilon \int_{\Omega} A^{\epsilon}\left(\nabla+2 i \pi \frac{\theta_{0}}{\epsilon}\right)\left(\frac{\partial \bar{\phi}}{\partial x_{k}} w_{\epsilon}\right) \cdot\left(\nabla-2 i \pi \frac{\theta_{0}}{\epsilon}\right) \bar{\zeta}_{k}^{\epsilon} \\
& -\int_{\Omega} A^{\epsilon} e_{k} \frac{\partial \bar{\phi}}{\partial x_{k}} w_{\epsilon} \cdot\left(\nabla-2 i \pi \frac{\theta_{0}}{\epsilon}\right) \bar{\psi}_{1}^{\epsilon} \\
& +\int_{\Omega} A^{\epsilon}\left(\nabla+2 i \pi \frac{\theta_{0}}{\epsilon}\right)\left(\frac{\partial \bar{\phi}}{\partial x_{k}} w_{\epsilon}\right) \cdot e_{k} \bar{\psi}_{1}^{\epsilon} \\
& -\int_{\Omega} A^{\epsilon} w_{\epsilon} \nabla \frac{\partial \bar{\phi}}{\partial x_{k}} \cdot e_{k} \bar{\psi}_{1}^{\epsilon} \\
& -\int_{\Omega} A^{\epsilon} w_{\epsilon} \nabla \frac{\partial \bar{\phi}}{\partial x_{k}} \cdot\left(\epsilon \nabla-2 i \pi \theta_{0}\right) \bar{\zeta}_{k}^{\epsilon} \\
& +\int_{\Omega} A^{\epsilon} \bar{\zeta}_{k}^{\epsilon}\left(\epsilon \nabla+2 i \pi \theta_{0}\right) w_{\epsilon} \cdot \nabla \frac{\partial \bar{\phi}}{\partial x_{k}}
\end{aligned}
$$

Now, for any smooth compactly supported test function $\Phi$ from $\Omega$ into $\mathbb{C}^{K}$, we deduce from the definition of $\psi_{1}$ that

$$
\int_{\Omega} A^{\epsilon}\left(\nabla+2 i \pi \frac{\theta_{0}}{\epsilon}\right) \psi_{1}^{\epsilon} \cdot\left(\nabla-2 i \pi \frac{\theta_{0}}{\epsilon}\right) \bar{\Phi}+\frac{1}{\epsilon^{2}} \int_{\Omega}\left(c^{\epsilon}-\lambda_{1}\left(\theta_{0}\right)\right) \psi_{1}^{\epsilon} \cdot \bar{\Phi}=0,
$$

and from the definition of $\zeta_{k}$

$$
\begin{gathered}
\int_{\Omega} A^{\epsilon}\left(\nabla+2 i \pi \frac{\theta_{0}}{\epsilon}\right) \zeta_{k}^{\epsilon} \cdot\left(\nabla-2 i \pi \frac{\theta_{0}}{\epsilon}\right) \bar{\Phi}+\frac{1}{\epsilon^{2}} \int_{\Omega}\left(c^{\epsilon}-\lambda_{1}\left(\theta_{0}\right)\right) \zeta_{k}^{\epsilon} \cdot \bar{\Phi}= \\
\epsilon^{-1} \int_{\Omega} A^{\epsilon}\left(\nabla+2 i \pi \frac{\theta_{0}}{\epsilon}\right) \psi_{1}^{\epsilon} \cdot e_{k} \bar{\Phi}-\epsilon^{-1} \int_{\Omega} A^{\epsilon} e_{k} \psi_{1}^{\epsilon} \cdot\left(\nabla-2 i \pi \frac{\theta_{0}}{\epsilon}\right) \bar{\Phi}
\end{gathered}
$$

Combining (48) with the potential term, we easily check that the first line of its right hand side cancels out because of (49) with $\Phi=\phi \bar{w}_{\epsilon}$, and the next three lines cancel out because of 50 with $\Phi=\frac{\partial \phi}{\partial x_{k}} \bar{w}_{\epsilon}$. On the other hand, we can pass to the limit in the three last terms of (48). Finally, (19) 
multiplied by $\bar{\Psi}_{\epsilon}$ yields after simplification

$$
\begin{aligned}
& \int_{\Omega} u_{\epsilon}^{0} \cdot \bar{\Psi}_{\epsilon}(t=0) d x-\int_{0}^{T} \int_{\Omega} w_{\epsilon} \cdot\left(\bar{\psi}_{1}^{\epsilon} \frac{\partial \bar{\phi}}{\partial t}+\epsilon \frac{\partial^{2} \bar{\phi}}{\partial x_{k} \partial t} \bar{\zeta}_{k}^{\epsilon}\right) d t d x \\
& -\int_{0}^{T} \int_{\Omega} A^{\epsilon} w_{\epsilon} \nabla \frac{\partial \bar{\phi}}{\partial x_{k}} \cdot e_{k} \bar{\psi}_{1}^{\epsilon} d t d x \\
& -\int_{0}^{T} \int_{\Omega} A^{\epsilon} w_{\epsilon} \nabla \frac{\partial \bar{\phi}}{\partial x_{k}} \cdot\left(\epsilon \nabla-2 i \pi \theta_{0}\right) \bar{\zeta}_{k}^{\epsilon} d t d x \\
& +\int_{0}^{T} \int_{\Omega} A^{\epsilon} \bar{\zeta}_{k}^{\epsilon}\left(\epsilon \nabla+2 i \pi \theta_{0}\right) w_{\epsilon} \cdot \nabla \frac{\partial \bar{\phi}}{\partial x_{k}} d t d x \\
& +\int_{0}^{T} \int_{\Omega} d^{\epsilon} w_{\epsilon} \cdot \bar{\Psi}_{\epsilon} d t d x
\end{aligned}
$$

Passing to the two-scale limit in each term of (51) gives

$$
\begin{aligned}
& \int_{\Omega} \int_{\mathbb{T}^{N}} \psi_{1} v^{0} \cdot \bar{\psi}_{1} \bar{\phi}(t=0) d x d y-\int_{0}^{T} \int_{\Omega} \int_{\mathbb{T}^{N}} \psi_{1} v \cdot \bar{\psi}_{1} \frac{\partial \bar{\phi}}{\partial t} d t d x d y \\
& -\int_{0}^{T} \int_{\Omega} \int_{\mathbb{T}^{N}} A \psi_{1} v \nabla \frac{\partial \bar{\phi}}{\partial x_{k}} \cdot e_{k} \bar{\psi}_{1} d t d x d y \\
& -\int_{0}^{T} \int_{\Omega} \int_{\mathbb{T}^{N}} A \psi_{1} v \nabla \frac{\partial \bar{\phi}}{\partial x_{k}} \cdot\left(\nabla_{y}-2 i \pi \theta_{0}\right) \bar{\zeta}_{k} d t d x d y \\
& +\int_{0}^{T} \int_{\Omega} \int_{\mathbb{T}^{N}} A \bar{\zeta}_{k}\left(\nabla_{y}+2 i \pi \theta_{0}\right) \psi_{1} v \cdot \nabla \frac{\partial \bar{\phi}}{\partial x_{k}} d t d x d y \\
& +\int_{0}^{T} \int_{\Omega} \int_{\mathbb{T}^{N}} d \psi_{1} v \cdot \bar{\psi}_{1} \bar{\phi} d t d x d y
\end{aligned}
$$$$
=0 \text {. }
$$

Recalling the normalization $\int_{\mathbb{T}^{N}}\left|\psi_{1}\right|^{2} d y=1$, and introducing

$$
\begin{aligned}
A_{j k}^{*}=\int_{\mathbb{T}^{N}} & \left(A \psi_{1} e_{j} \cdot e_{k} \bar{\psi}_{1}+A \psi_{1} e_{k} \cdot e_{j} \bar{\psi}_{1}\right. \\
& +A \psi_{1} e_{j} \cdot\left(\nabla_{y}-2 i \pi \theta_{0}\right) \bar{\zeta}_{k}+A \psi_{1} e_{k} \cdot\left(\nabla_{y}-2 i \pi \theta_{0}\right) \bar{\zeta}_{j} \\
& \left.-A \bar{\zeta}_{k}\left(\nabla_{y}+2 i \pi \theta_{0}\right) \psi_{1} \cdot e_{j}-A \bar{\zeta}_{j}\left(\nabla_{y}+2 i \pi \theta_{0}\right) \psi_{1} \cdot e_{k}\right) d y
\end{aligned}
$$

and $d^{*}(x)=\int_{\mathbb{T}^{N}} d(x, y) \psi_{1}(y) \cdot \bar{\psi}_{1}(y) d y, 52$ is equivalent to

$$
\int_{\Omega} v^{0} \bar{\phi}(0) d x-\int_{0}^{T} \int_{\Omega}\left(v \frac{\partial \bar{\phi}}{\partial t}+A^{*} v \cdot \nabla \nabla \bar{\phi}-d^{*}(x) v \bar{\phi}\right) d t d x=0
$$


which is a very weak form of the homogenized equation (24). Note, however, that we can not recover the Dirichlet boundary condition from (52). To this end we shall use the compactness Lemma 4.2 below which was not required so far (and which holds true for functions depending on time, as claimed in Remark 4.3). Since, by the parabolic energy estimate, assumption (55) is satisfied, we deduce that there exists a bounded scalar sequence $v_{\epsilon}$ in $L^{2}\left((0, T) ; H^{1}\left(\mathbb{R}^{N}\right)\right)$ such that

$$
u_{\epsilon}(t, x)=\psi_{1}\left(\frac{x}{\epsilon}, \theta_{0}\right) e^{2 i \pi \frac{\theta_{0} \cdot x}{\epsilon}} v_{\epsilon}(t, x)+r_{\epsilon}(t, x),
$$

and $\lim _{\epsilon \rightarrow 0}\left\|r_{\epsilon}\right\|_{L^{2}((0, T) \times \omega)^{K}}=0$ for any compact set $\omega \subset \mathbb{R}^{N}$. Up to a subsequence, $v_{\epsilon}$ converges weakly to a limit $v$ in $L^{2}\left((0, T) ; H^{1}\left(\mathbb{R}^{N}\right)\right)$, which necessarily coincides with the two-scale limit obtained in 46). If the compact set $\omega$ lies outside $\Omega$, i.e. $\omega \subset\left(\mathbb{R}^{N} \backslash \Omega\right)$, we deduce from (54) that

$$
\psi_{1}\left(\frac{x}{\epsilon}, \theta_{0}\right) v_{\epsilon}(t, x)=-r_{\epsilon}(t, x) \quad \text { in } \omega \times(0, T),
$$

and since $\psi_{1}$ is normalized, we obtain

$$
\left\|r_{\epsilon}\right\|_{L^{2}((0, T) \times \omega)^{K}}^{2}=\int_{\omega}\left|\psi_{1}\left(\frac{x}{\epsilon}, \theta_{0}\right)\right|^{2}\left|v_{\epsilon}(x)\right|^{2} d x \rightarrow \int_{\omega}|v(x)|^{2} d x=0 .
$$

Therefore, we deduce that $v=0$ in any compact set $\omega$ outside from $\Omega$. This implies that $v$ belongs to $H_{0}^{1}(\Omega)$.

The compatibility condition of equation 15 for the second derivative of $\psi_{1}$ yields that the matrix $A^{*}$, defined by (53), is indeed equal to $\frac{1}{4 \pi^{2}} \nabla_{\theta} \nabla_{\theta} \lambda_{1}\left(\theta_{0}\right)$, and thus is real, symmetric, positive definite by assumption (9). Therefore, the homogenized problem (24) is well posed. By uniqueness of the solution of the homogenized problem (24), we deduce that the entire sequence $v_{\epsilon}$ converges to $v$ (which is a real-valued function).

Remark 4.1 As usual in periodic homogenization, the choice of the test function $\Psi_{\epsilon}$, defined by (47), is dictated by the formal two-scale asymptotic expansion that can be obtained for the solution $u_{\epsilon}$ of $(17)$. Indeed, if one admits that the ansatz of $u_{\epsilon}$ starts with the following two exponential terms (which is not obvious a priori!), then a simple and formal computation shows that

$$
u_{\epsilon}(t, x) \approx e^{-\frac{\lambda_{1}\left(\theta_{0}\right) t}{\epsilon^{2}}} e^{2 i \pi \frac{\theta_{0} \cdot x}{\epsilon}}\left(\psi_{1}\left(\frac{x}{\epsilon}, \theta_{0}\right) v(t, x)+\epsilon \sum_{k=1}^{N} \frac{\partial v}{\partial x_{k}}(t, x) \zeta_{k}\left(\frac{x}{\epsilon}\right)\right),
$$

where $v$ is the homogenized solution of (24). 
Lemma 4.2 Let $u_{\epsilon}$ be a bounded sequence in $L^{2}\left(\mathbb{R}^{N}\right)^{K}$. Assume that there exists a finite constant $C$ such that

$$
\int_{\mathbb{R}^{N}}\left(A\left(\frac{x}{\epsilon}\right) \nabla u_{\epsilon} \cdot \nabla u_{\epsilon}+\frac{c\left(\frac{x}{\epsilon}\right)-\lambda_{1}\left(\theta_{0}\right)}{\epsilon^{2}} u_{\epsilon} \cdot u_{\epsilon}\right) d x \leq C .
$$

Then, under assumption (9),

$$
u_{\epsilon}(x)=\psi_{1}\left(\frac{x}{\epsilon}, \theta_{0}\right) e^{2 i \pi \frac{\theta_{0} \cdot x}{\epsilon}} v_{\epsilon}(x)+r_{\epsilon}(x),
$$

where $v_{\epsilon}$ is a bounded scalar sequence in $H^{1}\left(\mathbb{R}^{N}\right)$ and $\lim _{\epsilon \rightarrow 0}\left\|r_{\epsilon}\right\|_{L^{2}(\omega)^{K}}=0$ for any compact set $\omega \subset \mathbb{R}^{N}$.

Remark 4.3 If we consider functions depending on time, Lemma 4.2 is easily extended as follows. Assuming that $u_{\epsilon}$ is a bounded sequence in $L^{2}((0, T) \times$ $\left.\mathbb{R}^{N}\right)^{K}$ and that (55) holds true when integrated on the time interval $(0, T)$, the decomposition (56) is still valid with $v_{\epsilon}$ bounded in $L^{2}\left((0, T) ; H^{1}\left(\mathbb{R}^{N}\right)\right)$ and $r_{\epsilon}$ converging strongly to 0 in $L^{2}\left((0, T) ; L_{l o c}^{2}\left(\mathbb{R}^{N}\right)\right)$.

Remark 4.4 If the sequence $u_{\epsilon}$ further vanishes outside an open set $\Omega$, then we can obtain the representation (26) with $v_{\epsilon}$ uniformly bounded in $H_{0}^{1}(\Omega)$. Indeed, it is enough to project the function $v_{\epsilon} \in H^{1}\left(\mathbb{R}^{N}\right)$, given by Lemma 4.2, on $H_{0}^{1}(\Omega)$.

Proof. Our proof is in the spirit of the previous works [18, [16]. Applying the rescaled Bloch decomposition 16 to $u_{\epsilon}(x)$ with $\eta=\frac{\theta-\theta_{0}}{\epsilon}$, we have

$$
u_{\epsilon}(x)=\sum_{k \geq 1} \int_{\epsilon^{-1} \mathbb{T}^{N}} \alpha_{k}^{\epsilon}(\eta) \psi_{k}\left(\frac{x}{\epsilon}, \theta_{0}+\epsilon \eta\right) e^{2 i \pi \eta \cdot x} e^{2 i \pi \frac{\theta_{0} \cdot x}{\epsilon}} d \eta
$$

and

$$
\begin{aligned}
& \int_{\mathbb{R}^{N}}\left(A\left(\frac{x}{\epsilon}\right) \nabla u_{\epsilon} \cdot \nabla u_{\epsilon}+\frac{c\left(\frac{x}{\epsilon}\right)-\lambda_{1}\left(\theta_{0}\right)}{\epsilon^{2}} u_{\epsilon} \cdot u_{\epsilon}\right) d x \\
& =\epsilon^{-2} \sum_{k \geq 1} \int_{\epsilon^{-1} \mathbb{T}^{N}}\left|\alpha_{k}^{\epsilon}(\eta)\right|^{2}\left(\lambda_{k}\left(\theta_{0}+\epsilon \eta\right)-\lambda_{1}\left(\theta_{0}\right)\right) d \eta .
\end{aligned}
$$

Since $\lambda_{k}(\theta)-\lambda_{1}\left(\theta_{0}\right) \geq 0$ and, for $k \geq 2, \lambda_{k}(\theta)-\lambda_{1}\left(\theta_{0}\right) \geq C>0$, we deduce from the bound (55) that

$$
\sum_{k \geq 2} \int_{\epsilon^{-1} \mathbb{T}^{N}}\left|\alpha_{k}^{\epsilon}(\eta)\right|^{2} d \eta \leq C \epsilon^{2}
$$


For $k=1$, by assumption (9) there exists $C>0$ such that

$$
\lambda_{1}(\theta)-\lambda_{1}\left(\theta_{0}\right) \geq C\left|\theta-\theta_{0}\right|^{2} \quad \forall \theta \in \mathbb{T}^{N},
$$

and thus 55 implies

$$
\int_{\epsilon^{-1} \mathbb{T}^{N}}|\eta|^{2}\left|\alpha_{1}^{\epsilon}(\eta)\right|^{2} d \eta \leq C
$$

Extending $\alpha_{1}^{\epsilon}(\eta)$ by zero outside $\epsilon^{-1} \mathbb{T}^{N}$, and using the inverse Fourier transform, we deduce that the scalar sequence $v_{\epsilon}$, defined by

$$
v_{\epsilon}(x)=\int_{\mathbb{R}^{N}} \alpha_{1}^{\epsilon}(\eta) e^{2 i \pi \eta \cdot x} d \eta
$$

is bounded in $H^{1}\left(\mathbb{R}^{N}\right)$.

Introducing a parameter $0<q<1$ (to be chosen later) we define a cut-off of $v_{\epsilon}$ by

$$
\tilde{v}_{\epsilon}=\int_{|\eta|<\epsilon^{-q}} \alpha_{1}^{\epsilon}(\eta) e^{2 i \pi \eta \cdot x} d \eta .
$$

The difference between $v_{\epsilon}$ and $\tilde{v}_{\epsilon}$ is small since

$$
\left\|\tilde{v}_{\epsilon}-v_{\epsilon}\right\|_{L^{2}\left(\mathbb{R}^{N}\right)}^{2}=\int_{|\eta|>\epsilon^{-q}}\left|\alpha_{1}^{\epsilon}(\eta)\right|^{2} d \eta \leq \epsilon^{2 q} \int_{\mathbb{R}^{N}}|\eta|^{2}\left|\alpha_{1}^{\epsilon}(\eta)\right|^{2} d \eta \leq C \epsilon^{2 q}
$$

Similarly we have

$$
\int_{\epsilon^{-1} \mathbb{T}^{N}} \alpha_{1}^{\epsilon}(\eta) \psi_{1}\left(\frac{x}{\epsilon}, \theta_{0}+\epsilon \eta\right) e^{2 i \pi \eta \cdot x} e^{2 i \pi \frac{\theta_{0} \cdot x}{\epsilon}} d \eta=\int_{|\eta|<\epsilon^{-q}} \alpha_{1}^{\epsilon}(\eta) \psi_{1}\left(\frac{x}{\epsilon}, \theta_{0}+\epsilon \eta\right) e^{2 i \pi \eta \cdot x} e^{2 i \pi \frac{\theta_{0} \cdot x}{\epsilon}} d \eta+t_{\epsilon}(x),
$$

where $t_{\epsilon}$ is small, i.e.

$$
\left\|t_{\epsilon}\right\|_{L^{2}\left(\mathbb{R}^{N}\right)}^{2}=\int_{\eta \in \epsilon^{-1} \mathbb{T}^{N},|\eta|>\epsilon^{-q}}\left|\alpha_{1}^{\epsilon}(\eta)\right|^{2} d \eta \leq \epsilon^{2 q} \int_{\epsilon^{-1} \mathbb{T}^{N}}|\eta|^{2}\left|\alpha_{1}^{\epsilon}(\eta)\right|^{2} d \eta \leq C \epsilon^{2 q} .
$$

By the analycity of the first eigencouple of (8), there exists a periodic function $\chi \in L^{2}\left(\mathbb{T}^{N}\right)$ such that

$$
\left|\psi_{1}(y, \theta)-\psi_{1}\left(y, \theta_{0}\right)\right| \leq\left|\theta-\theta_{0}\right| \chi(y) \quad \forall y \in \mathbb{T}^{N}, \theta \in \mathbb{T}^{N} .
$$

Therefore, we have

$$
\int_{|\eta|<\epsilon^{-q}} \alpha_{1}^{\epsilon}(\eta) \psi_{1}\left(\frac{x}{\epsilon}, \theta_{0}+\epsilon \eta\right) e^{2 i \pi \eta \cdot x} e^{2 i \pi \frac{\theta_{0} \cdot x}{\epsilon}} d \eta=\psi_{1}\left(\frac{x}{\epsilon}, \theta_{0}\right) e^{2 i \pi \frac{\theta_{0} \cdot x}{\epsilon}} \tilde{v}_{\epsilon}(x)+s_{\epsilon}(x)
$$


where $s_{\epsilon}$ is small, i.e.

$$
\begin{aligned}
\left|s_{\epsilon}(x)\right| & \leq \chi\left(\frac{x}{\epsilon}\right) \int_{|\eta|<\epsilon^{-q}} \epsilon|\eta|\left|\alpha_{1}^{\epsilon}(\eta)\right| d \eta \\
& \leq \epsilon \chi\left(\frac{x}{\epsilon}\right)\left(\int_{|\eta|<\epsilon^{-q}}|\eta|^{2}\left|\alpha_{1}^{\epsilon}(\eta)\right|^{2} d \eta\right)^{1 / 2}\left(\int_{|\eta|<\epsilon^{-q}} d \eta\right)^{1 / 2} \\
& \leq C \epsilon^{1-\frac{N q}{2}} \chi\left(\frac{x}{\epsilon}\right) .
\end{aligned}
$$

Thus, we obtain that, for any compact set $\omega \subset \mathbb{R}^{N}$,

$$
\left\|s_{\epsilon}\right\|_{L^{2}(\omega)^{K}}^{2} \leq C|\omega| \epsilon^{1-\frac{N q}{2}} .
$$

(We can not obtain a uniform estimate on $\mathbb{R}^{N}$ since $s_{\epsilon}$ is not defined as a Bloch decomposition.) Collecting all the intermediate steps we deduce

$$
u_{\epsilon}(x)=\psi_{1}\left(\frac{x}{\epsilon}, \theta_{0}\right) e^{2 i \pi \frac{\theta_{0} \cdot x}{\epsilon}} \tilde{v}_{\epsilon}(x)+r_{\epsilon}(x)
$$

and $\left\|r_{\epsilon}\right\|_{L^{2}(\omega)^{K}} \leq C|\omega| \epsilon^{\frac{2}{N+2}}$ with the optimal value of $q$ equal to $2 /(N+2)$.

Remark 4.5 If we remove from assumption (9) the positive definite character of the Hessian matrix $\nabla_{\theta} \nabla_{\theta} \lambda_{1}\left(\theta_{0}\right)$, we can still obtained an homogenization result, weaker than Theorem 3.2. Indeed, the same proof shows that $w_{\epsilon}$ two-scales converges, up to a subsequence, to $\psi_{1}\left(y, \theta_{0}\right) v(t, x)$ where $v$ is a solution of the homogenized equation (24) with a possibly degenerate matrix $A^{*}$ (which is nevertheless always non-negative because $\theta_{0}$ is a minimum point). However, Lemma 4.2 holds true only if $\nabla_{\theta} \nabla_{\theta} \lambda_{1}\left(\theta_{0}\right)$ is positive definite. Thus, we can not recover the Dirichlet boundary condition, neither can we obtain the uniqueness of the homogenized solution and the convergence of the entire sequence $w_{\epsilon}$.

Remark 4.6 If we remove from assumption (9) the fact that the minimum point $\theta_{0}$ of $\lambda_{1}(\theta)$ is unique, then we can also prove a weaker version of Theorem 3.2. For each minimum and associated Hessian matrix $\nabla_{\theta} \nabla_{\theta} \lambda_{1}$, we can extract a subsequence such that $w_{\epsilon}$ two-scales converges $\psi_{1}\left(y, \theta_{0}\right) v(t, x)$ where $v$ is a solution of the homogenized equation (24). However, since Lemma 4.2 does not hold true in this case, we can not recover the Dirichlet boundary condition. Nevertheless, if $\Omega=\mathbb{R}^{N}$ and $\nabla_{\theta} \nabla_{\theta} \lambda_{1}$ is positive definite, 
we do not need any boundary condition to obtain the unique solvability of the homogenized equation. Thus, in such a case, the entire sequence $w_{\epsilon}$ is

converging. Recall that $w_{\epsilon}=e^{\frac{\lambda_{1}\left(\theta_{0}\right) t}{\epsilon^{2}}} e^{-2 i \pi \frac{\theta_{0} \cdot x}{\epsilon}} u_{\epsilon}$, so that for different minima we have different values of $\theta_{0}$, thus different sequences $w_{\epsilon}$, and eventually different homogenized problems. If the initial condition is a superposition of well-prepared initial data for each minimum point $\theta_{0}$, then, by linearity, we can decompose the solution in a superposition of elementary solutions, each of them converging to its own homogenized limit depending on $\theta_{0}$.

\section{Proofs for the spectral problem}

This section is devoted to the proof of Theorem 3.6. For simplicity reasons, we first prove the analogue of this result in the whole space, $\Omega=\mathbb{R}^{N}$, with, on top of (9), the additional assumption

$$
\lim _{|x| \rightarrow+\infty} \sum_{\alpha, \beta=1}^{K} d_{\alpha \beta}(x, y) \eta_{\alpha} \eta_{\beta}=+\infty \text { uniformly in } y \in \mathbb{T}^{N}, \eta \in\{|\eta|=1\} .
$$

Under this assumption, it is well-known that the following spectral problem

$$
-\operatorname{div}\left(A\left(\frac{x}{\epsilon}\right) \nabla u_{\epsilon}\right)+\epsilon^{-2} c\left(\frac{x}{\epsilon}\right) u_{\epsilon}+d\left(x, \frac{x}{\epsilon}\right) u_{\epsilon}=\lambda_{\epsilon} u_{\epsilon} \quad \text { in } \mathbb{R}^{N}
$$

has a discrete spectrum in $L^{2}\left(\mathbb{R}^{N}\right)^{K}, \lambda_{1}^{\epsilon} \leq \lambda_{2}^{\epsilon} \leq \ldots \leq \lambda_{n}^{\epsilon} \ldots \rightarrow+\infty$, with corresponding orthonormal eigenfunctions denoted by $u_{1}^{\epsilon}(x), u_{2}^{\epsilon}(x), \ldots$. Similarly, since $\lim _{|x| \rightarrow+\infty} d^{*}(x)=+\infty$, the homogenized problem (27) has a discrete spectrum, $\mu_{1}, \mu_{2}, \ldots, \mu_{n}, \ldots \rightarrow+\infty$, with corresponding orthonormal eigenfunctions denoted by $v_{1}(x), v_{2}(x), \ldots$.

Lemma 5.1 There exists a constant $C>0$, which does not depend on $\epsilon$, such that

$$
\frac{\lambda_{1}\left(\theta_{0}\right)}{\epsilon^{2}}+C \leq \lambda_{1}^{\epsilon} \leq \frac{\lambda_{1}\left(\theta_{0}\right)}{\epsilon^{2}}+\mu_{1}+o(1),
$$

where o(1) vanishes as $\epsilon \rightarrow 0$.

Proof. We assume that $v_{1}$ is smooth enough, which is not a restriction since the general case can be reduced to this one by means of approximation. We write down the variational formulation

$$
\lambda_{1}^{\epsilon}=\min _{\|u\|_{L^{2}\left(\mathbb{R}^{N}\right)^{K}=1}} \int_{\mathbb{R}^{N}}\left(A^{\epsilon} \nabla u \cdot \nabla \bar{u}+\left(\epsilon^{-2} c^{\epsilon}+d^{\epsilon}\right) u \cdot \bar{u}\right) d x
$$


and substitute a test function of the form

$$
U^{\epsilon}=\gamma_{\epsilon} e^{2 i \pi \frac{\theta_{0} \cdot x}{\epsilon}}\left(\psi_{1}\left(\frac{x}{\epsilon}, \theta_{0}\right) v_{1}(x)+\epsilon \sum_{k=1}^{N} \frac{\partial v_{1}}{\partial x_{k}}(x) \zeta_{k}\left(\frac{x}{\epsilon}\right)\right),
$$

where $\zeta_{k}$ is the solution of (14) and $\gamma_{\epsilon}$ is a normalization constant chosen in such a way that $\left\|U^{\epsilon}\right\|_{L^{2}\left(\mathbb{R}^{N}\right)^{K}}=1$. Since $\psi_{1}$ and $\zeta_{k}$ are periodic functions, and due to the assumptions on $v_{1}$, we have $\lim _{\epsilon \rightarrow 0} \gamma_{\epsilon}=1$. In view of $10 p$ and (14), after simple rearrangements we obtain

$$
\begin{gathered}
\lambda_{1}^{\epsilon} \leq \frac{\lambda_{1}\left(\theta_{0}\right)}{\epsilon^{2}}+o(1)+\gamma_{\epsilon}^{2} \int_{\mathbb{R}^{N}} A_{\alpha \beta, k l}^{\epsilon} \psi_{1, \alpha}^{\epsilon} \bar{\psi}_{1, \beta}^{\epsilon} \frac{\partial v_{1}}{\partial x_{k}} \frac{\partial v_{1}}{\partial x_{l}} d x \\
+\gamma_{\epsilon}^{2} \int_{\mathbb{R}^{N}}\left\{\bar{\psi}_{1, \alpha}^{\epsilon} A_{\alpha \beta, m l}^{\epsilon}\left(\frac{\partial}{\partial y_{m}}+2 i \pi \theta_{0, m}\right) \zeta_{k, \beta}^{\epsilon} \frac{\partial v_{1}}{\partial x_{l}} \frac{\partial v_{1}}{\partial x_{k}}\right. \\
\left.+\psi_{1, \alpha}^{\epsilon}\left(\frac{\partial}{\partial y_{k}}-2 i \pi \theta_{0, k}\right)\left(A_{\alpha \beta, k m}^{\epsilon} \bar{\zeta}_{l, \beta}^{\epsilon}\right) \frac{\partial v_{1}}{\partial x_{m}} \frac{\partial v_{1}}{\partial x_{l}}\right\} d x \\
+\gamma_{\epsilon}^{2} \int_{\mathbb{R}^{N}} \bar{\psi}_{1, \alpha}^{\epsilon} \psi_{1, \beta}^{\epsilon} d_{\alpha \beta}^{\epsilon}\left|v_{1}\right|^{2} d x+\epsilon^{2} \gamma_{\epsilon}^{2} \int_{\mathbb{R}^{N}} d^{\epsilon}\left(\zeta^{\epsilon} \nabla v_{1}\right) \cdot\left(\bar{\zeta}^{\epsilon} \nabla v_{1}\right) d x \\
+2 \epsilon \gamma_{\epsilon}^{2} \mathcal{R}\left(\int_{\mathbb{R}^{N}}\left(\bar{\psi}_{1}^{\epsilon} \zeta^{\epsilon} A^{\epsilon} \nabla \nabla v_{1} v_{1}+d^{\epsilon}\left(v_{1} \bar{\psi}_{1}^{\epsilon}\right) \cdot\left(\zeta^{\epsilon} \nabla v_{1}\right)\right) d x\right) .
\end{gathered}
$$

¿From the definitions of $A^{*}$ and $d^{*}$, we deduce the upper bound in (59). On the other hand, by using Lemma 2.4 we have

$$
\begin{gathered}
\min _{\|u\|_{L^{2}\left(\mathbb{R}^{N}\right)^{K}}=1} \int_{\mathbb{R}^{N}}\left(A^{\epsilon} \nabla u \cdot \nabla \bar{u}+\left(\epsilon^{-2} c^{\epsilon}+d^{\epsilon}\right) u \cdot \bar{u}\right) d x \geq \\
\frac{\lambda_{1}\left(\theta_{0}\right)}{\epsilon^{2}}+\inf _{x \in \mathbb{R}^{N}, y \in \mathbb{T}^{N},|\eta|=1} d(x, y) \eta \cdot \eta
\end{gathered}
$$

which yields the desired lower bound.

Lemma 5.2 There exists a scalar sequence $v_{\epsilon}$ which is relatively compact in $L^{2}\left(\mathbb{R}^{N}\right)$ and such that

$$
u_{1}^{\epsilon}(x)=v_{\epsilon}(x) \psi_{1}\left(\frac{x}{\epsilon}, \theta_{0}\right) e^{2 i \pi \frac{\theta_{0} \cdot x}{\epsilon}}+r_{\epsilon}(x)
$$


where

$$
\lim _{\epsilon \rightarrow 0}\left\|r_{\epsilon}\right\|_{L^{2}\left(\mathbb{R}^{N}\right)^{K}}=0, \quad\left\|v_{\epsilon}\right\|_{H^{1}\left(\mathbb{R}^{N}\right)} \leq C .
$$

Remark 5.3 As a consequence of Lemma 5.2 $v_{\epsilon}$ is almost normalized, i.e. $\left\|v_{\epsilon}\right\|_{L^{2}\left(\mathbb{R}^{N}\right)}=1+o(1)$.

Proof. ¿From Lemma 5.1 we deduce

$$
\frac{\lambda_{1}\left(\theta_{0}\right)}{\epsilon^{2}}+\int_{\mathbb{R}^{N}} d^{\epsilon} u_{1}^{\epsilon} \cdot \bar{u}_{1}^{\epsilon} d x \leq \lambda_{1}^{\epsilon} \leq \frac{\lambda_{1}\left(\theta_{0}\right)}{\epsilon^{2}}+\mu_{1}+o(1),
$$

which implies that

$$
\int_{\mathbb{R}^{N}} d^{\epsilon} u_{1}^{\epsilon} \cdot \bar{u}_{1}^{\epsilon} d x \leq C
$$

Then, the first part of Lemma 5.2 is a consequence of Lemma 4.2 since

$\int_{\mathbb{R}^{N}}\left(A^{\epsilon} \nabla u_{1}^{\epsilon} \cdot \nabla \bar{u}_{1}^{\epsilon}+\epsilon^{-2}\left(c^{\epsilon}-\lambda_{1}\left(\theta_{0}\right)\right) u_{1}^{\epsilon} \cdot \bar{u}_{1}^{\epsilon}\right) d x=\lambda_{1}^{\epsilon}-\frac{\lambda_{1}\left(\theta_{0}\right)}{\epsilon^{2}}-\int_{\mathbb{R}^{N}} d^{\epsilon} u_{1}^{\epsilon} \cdot \bar{u}_{1}^{\epsilon} d x \leq C$.

To obtain the relative compactness of $v_{\epsilon}$ in $L^{2}\left(\mathbb{R}^{N}\right)$ we deduce from 63 and the growth condition on $d^{\epsilon}(x)$ at infinity, that for each $\delta>0$ there is $R=R(\delta)$ such that

$$
\left\|u_{1}^{\epsilon}\right\|_{L^{2}(\{x:|x| \geq R\})^{K}}<\delta .
$$

Since $v_{\epsilon}$ is bounded in $H^{1}\left(\mathbb{R}^{N}\right)$, we can replace $\left(\psi_{1}\right)^{2}$ by its positive average in (64) and thus obtain

$$
\left\|v_{\epsilon}\right\|_{L^{2}(\{x:|x| \geq R\})}<\delta
$$

which implies the relative compactness.

Proof of Theorem 3.6 for $\Omega=\mathbb{R}^{N}$.

We focus on the first eigenfunction, $k=1$. For $k>1$ a similar proof holds true.

By Lemma 5.2 the family $v_{\epsilon}$ is relatively compact in $L^{2}\left(\mathbb{R}^{N}\right)$. Moreover, any limit point $v^{0}$ of a converging subsequence, satisfies the relation $\left\|v^{0}\right\|_{L^{2}\left(\mathbb{R}^{N}\right)}=1$. By Lemma 5.1 we can also extract a subsequence such that $\lambda_{1}^{\epsilon}-\frac{\lambda_{1}\left(\theta_{0}\right)}{\epsilon^{2}}$ converges to a limit $\bar{\mu}$. According to 59

$$
C \leq \bar{\mu} \leq \mu_{1} .
$$


The proof is now very similar to that of Theorem 3.2 (see Section 4). Up to another subsequence, $e^{-2 \pi i x \cdot \theta_{0} / \epsilon} u_{1}^{\epsilon}(x)$ two-scale converges to a limit $u_{1}^{0}(x, y)$ and $\epsilon \nabla\left(e^{-2 \pi i x \cdot \theta_{0} / \epsilon} u_{1}^{\epsilon}\right)$ two-scale converges to $\nabla_{y} u_{1}^{0}(x, y)$. As in the first step of the proof of Theorem 3.2 , one can easily show that

$$
u_{1}^{0}(x, y)=v^{0}(x) \psi_{1}\left(y, \theta_{0}\right)
$$

where $v^{0}$ is a limit point of $v_{\epsilon}$. To find the equation satisfied by $v^{0}$, we proceed as in the second step of the proof of Theorem 3.2. We multiply (58) by the test function

$$
\Psi_{\epsilon}(x)=e^{2 i \pi \frac{\theta_{0} \cdot x}{\epsilon}}\left(\psi_{1}\left(\frac{x}{\epsilon}, \theta_{0}\right) \phi(x)+\epsilon \sum_{k=1}^{N} \frac{\partial \phi}{\partial x_{k}}(x) \zeta_{k}\left(\frac{x}{\epsilon}\right)\right),
$$

where $\phi$ is smooth with compact support. This yields

$$
\begin{gathered}
\int_{\mathbb{R}^{N}} A^{\epsilon}(x) \nabla u_{1}^{\epsilon}(x) \nabla \bar{\Psi}_{\epsilon}(x) d x+\int_{\mathbb{R}^{N}} \frac{c^{\epsilon}(x)-\lambda_{1}\left(\theta_{0}\right)}{\epsilon^{2}} u_{1}^{\epsilon}(x) \cdot \bar{\Psi}_{\epsilon}(x) d x+ \\
\quad+\int_{\mathbb{R}^{N}} d^{\epsilon}(x) u_{1}^{\epsilon}(x) \cdot \bar{\Psi}_{\epsilon}(x)=\frac{\lambda_{1}^{\epsilon}-\lambda_{1}\left(\theta_{0}\right)}{\epsilon^{2}} \int_{\mathbb{R}^{N}} u_{1}^{\epsilon}(x) \cdot \bar{\Psi}_{\epsilon}(x) .
\end{gathered}
$$

As before, using (8) and (14), we can pass to the two scale limit to obtain

$$
\int_{\mathbb{R}^{N}}\left(A^{*} v^{0} \cdot \nabla \nabla \bar{\phi}+d^{*}(x) v^{0} \bar{\phi}\right) d x=\bar{\mu} \int_{\mathbb{R}^{N}} v^{0} \varphi d x
$$

which is a weak variational formulation of

$$
-A^{*} \cdot \nabla \nabla v^{0}+d^{*} v^{0}=\bar{\mu} v^{0} \quad \text { in } \quad \mathbb{R}^{N} .
$$

Since $v^{0} \neq 0$ and $\bar{\mu} \leq \mu_{1}$, we necessarily have

$$
\bar{\mu}=\mu_{1},
$$

and $v^{0}$ is an eigenfunction of (27) associated with $\mu_{1}$. If $\mu_{1}$ is simple, up to a convenient renormalization, the entire sequence $u_{1}^{\epsilon}$ is converging (and not merely a subsequence).

\section{Proof of Theorem 3.6 for a bounded open set $\Omega$.}


We merely outline the differences with the case $\Omega=\mathbb{R}^{N}$. In the proof of Lemma 5.1, in order to get an upper bound for $\lambda_{1}^{\epsilon}$, we can not use the test function (61) because it does not satisfy the Dirichlet boundary condition. Therefore we replace $v_{1}$ by a convenient approximation. For each small $\delta>0$, we introduce a smooth and compactly supported in $\Omega$ function $w^{\delta}$, such that $\left\|w^{\delta}\right\|_{L^{2}(\Omega)}=1$ and

$$
\int_{\Omega}\left(A^{*} \nabla w^{\delta} \cdot \nabla \bar{w}^{\delta}+d^{*}(x)\left|w^{\delta}\right|^{2}\right) d x<\mu_{1}+\delta .
$$

Substituting in the Rayleigh quotient defining $\lambda_{1}^{\epsilon}$ a test function of the form

$$
U_{\delta}^{\epsilon}=\gamma_{\epsilon} e^{2 i \pi \frac{\theta_{0} \cdot x}{\epsilon}}\left(\psi_{1}\left(\frac{x}{\epsilon}, \theta_{0}\right) w^{\delta}(x)+\epsilon \sum_{k=1}^{N} \frac{\partial w^{\delta}}{\partial x_{k}}(x) \zeta_{k}\left(\frac{x}{\epsilon}\right)\right),
$$

in the same manner we get the estimate

$$
\lambda_{1}^{\epsilon} \leq \frac{\lambda_{1}\left(\theta_{0}\right)}{\epsilon^{2}}+\mu_{1}+\delta+o(1),
$$

where $o(1)$ vanishes as $\epsilon \rightarrow 0$. Since $\delta$ is an arbitrary positive number, this yields the required upper bound. The lower bound is obtained as before, extending first $u_{1}^{\epsilon}$ to the whole space $\mathbb{R}^{N}$ by setting $u_{1}^{\epsilon}(x)=0$ for $x \in \mathbb{R}^{N} \backslash \Omega$, and using formula 16 .

Lemma 5.2 is unchanged, equation 66 is obtained as before, and the Dirichlet boundary condition for the limit $v^{0}$ is recovered as in the parabolic case (see the end of the proof of Theorem 3.2.).

\section{Proofs in the hyperbolic case}

We begin with proof of Theorem 3.7 when $\lambda_{1}\left(\theta_{0}\right)=0$. Actually, as soon as uniform a priori estimates are obtained for the solution of equation (28), the proof of convergence is completely similar to that of Theorem 3.2 in the parabolic case. Therefore, for the sake of brevity, we content ourselves in establishing those a priori estimates.

Lemma 6.1 Under the assumptions of Theorem 3.7 the solution $u_{\epsilon}$ of (28) satisfies

$$
\left\|u_{\epsilon}\right\|_{L^{\infty}\left((0, T) ; L^{2}(\Omega)^{K}\right)}+\epsilon\left\|\nabla u_{\epsilon}\right\|_{L^{2}((0, T) \times \Omega)^{N \times K}}+\left\|\frac{\partial u_{\epsilon}}{\partial t}\right\|_{L^{\infty}\left((0, T) ; L^{2}(\Omega)^{K}\right)} \leq C,
$$


where $C>0$ is a constant which does not depend on $\epsilon$. Furthermore, there exists a scalar sequence $v_{\epsilon}$, uniformly bounded in $L^{2}\left((0, T) ; H^{1}(\Omega)\right)$, such that

$$
u_{\epsilon}(t, x)=\psi_{1}\left(\frac{x}{\epsilon}, \theta_{0}\right) e^{2 i \pi \frac{\theta_{0} \cdot x}{\epsilon}} v_{\epsilon}(t, x)+r_{\epsilon}(t, x),
$$

where $r_{\epsilon}$ is a remainder term such that

$$
\lim _{\epsilon \rightarrow 0}\left\|r_{\epsilon}\right\|_{L^{2}((0, T) \times \omega)^{K}}=0 \text { for any compact set } \omega \subset \mathbb{R}^{N} .
$$

Proof. We multiply 28 by $\frac{\partial u_{\epsilon}}{\partial t}$ to obtain the usual energy estimate

$$
E_{\epsilon}(t)=E_{\epsilon}(0) \text { with } E_{\epsilon}(t)=\frac{1}{2} \int_{\Omega}\left(\left|\frac{\partial u_{\epsilon}}{\partial t}\right|^{2}+A^{\epsilon} \nabla u_{\epsilon} \cdot \nabla u_{\epsilon}+\frac{c^{\epsilon}}{\epsilon^{2}} u_{\epsilon} \cdot u_{\epsilon}\right) d x
$$

Since $\lambda_{1}\left(\theta_{0}\right)=0$, by using (8), a classical computation shows that

$$
E_{\epsilon}(0)=\frac{1}{2} \int_{\Omega}\left(\left|u_{\epsilon}^{1}\right|^{2}+A^{\epsilon}\left(\psi_{\epsilon}^{1} \otimes \nabla v^{0}\right) \cdot\left(\psi_{\epsilon}^{1} \otimes \nabla v^{0}\right)\right) d x
$$

which is uniformly bounded by assumption. Then, the Bloch wave analysis of Lemma 3.1 yields

$$
\int_{\Omega}\left(A^{\epsilon} \nabla u_{\epsilon} \cdot \nabla u_{\epsilon}+\frac{c^{\epsilon}}{\epsilon^{2}} u_{\epsilon} \cdot u_{\epsilon}\right) d x \geq 0 .
$$

Therefore, we deduce (68) from (71). To obtain $(69)$ and $(70)$ we use Lemma 4.2 since (71) implies that assumption (55) is satisfied.

We now turn to the proof of Theorem 3.8 when $\lambda_{1}\left(\theta_{0}\right)<0$. Once again the proof of convergence is very similar to that of Theorem 3.2 as soon as uniform a priori estimates are established (see [3] in the scalar case if necessary). Therefore, we restrict ourselves to obtaining a priori estimates for the rescaled hyperbolic system (37).

Lemma 6.2 Under the assumptions of Theorem 3.8 the solution $\tilde{u}_{\epsilon}$ of (37) satisfies

$$
\left\|\tilde{u}_{\epsilon}\right\|_{L^{\infty}\left((0, T) ; L^{2}(\Omega)^{K}\right)}+\epsilon\left\|\nabla \tilde{u}_{\epsilon}\right\|_{L^{2}((0, T) \times \Omega)^{N \times K}}+\epsilon\left\|\frac{\partial \tilde{u}_{\epsilon}}{\partial t}\right\|_{L^{2}((0, T) \times \Omega)^{K}} \leq C,
$$


where $C>0$ is a constant which does not depend on $\epsilon$. Furthermore, there exists a scalar sequence $v_{\epsilon}$, uniformly bounded in $L^{2}\left((0, T) ; H^{1}(\Omega)\right)$, such that

$$
\tilde{u}_{\epsilon}(t, x)=\psi_{1}\left(\frac{x}{\epsilon}, \theta_{0}\right) e^{2 i \pi \frac{\theta_{0} \cdot x}{\epsilon}} v_{\epsilon}(t, x)+r_{\epsilon}(t, x),
$$

where $r_{\epsilon}$ is a remainder term such that

$$
\lim _{\epsilon \rightarrow 0}\left\|r_{\epsilon}\right\|_{L^{2}((0, T) \times \omega)^{K}}=0 \text { for any compact set } \omega \subset \mathbb{R}^{N} .
$$

Proof. In a first step we multiply (35) by $\frac{\partial \tilde{u}_{\epsilon}}{\partial t}$ to obtain the usual energy estimate

$$
E_{\epsilon}(T)+2 \sqrt{-\lambda_{1}\left(\theta_{0}\right)} \int_{0}^{T} \int_{\Omega}\left|\frac{\partial \tilde{u}_{\epsilon}}{\partial t}\right|^{2} d x d t=E_{\epsilon}(0)
$$

with

$$
E_{\epsilon}(t)=\frac{1}{2} \int_{\Omega}\left(\epsilon^{2}\left|\frac{\partial \tilde{u}_{\epsilon}}{\partial t}\right|^{2}+A^{\epsilon} \nabla \tilde{u}_{\epsilon} \cdot \nabla \tilde{u}_{\epsilon}+\frac{c^{\epsilon}-\lambda_{1}\left(\theta_{0}\right)}{\epsilon^{2}} \tilde{u}_{\epsilon} \cdot \tilde{u}_{\epsilon}\right) d x .
$$

As in the proof of Lemma 6.1, using (8) yields

$$
\int_{\Omega}\left(A^{\epsilon} \nabla u_{\epsilon}^{0} \cdot \nabla u_{\epsilon}^{0}+\frac{c^{\epsilon}-\lambda_{1}\left(\theta_{0}\right)}{\epsilon^{2}} u_{\epsilon}^{0} \cdot u_{\epsilon}^{0}\right) d x=\int_{\Omega} A^{\epsilon}\left(\psi_{\epsilon}^{1} \otimes \nabla v^{0}\right) \cdot\left(\psi_{\epsilon}^{1} \otimes \nabla v^{0}\right) d x,
$$

which is however not sufficient to show that $E_{\epsilon}(0)$ is uniformly bounded. Indeed we have

$$
\frac{\partial \tilde{u}_{\epsilon}}{\partial t}(0)=u_{\epsilon}^{1}-\frac{\sqrt{-\lambda_{1}\left(\theta_{0}\right)}}{\epsilon^{2}} u_{\epsilon}^{0}
$$

which merely implies

$$
E_{\epsilon}(0) \leq C \epsilon^{-2} .
$$

Nevertheless, from the Bloch wave analysis of Lemma 3.1 we deduce

$$
\int_{\Omega}\left(A^{\epsilon} \nabla \tilde{u}_{\epsilon} \cdot \nabla \tilde{u}_{\epsilon}+\frac{c^{\epsilon}-\lambda_{1}\left(\theta_{0}\right)}{\epsilon^{2}} \tilde{u}_{\epsilon} \cdot \tilde{u}_{\epsilon}\right) d x \geq 0,
$$

which, combined with 75, yields

$$
\epsilon^{2}\left\|\frac{\partial \tilde{u}_{\epsilon}}{\partial t}\right\|_{L^{\infty}\left((0, T) ; L^{2}(\Omega)^{K}\right)}+\epsilon \sqrt{-\lambda_{1}\left(\theta_{0}\right)}\left\|\frac{\partial \tilde{u}_{\epsilon}}{\partial t}\right\|_{L^{2}((0, T) \times \Omega)^{K}} \leq C .
$$


In a second step we multiply (35) by $\tilde{u}_{\epsilon}$ to obtain a better energy estimate

$$
\begin{aligned}
& \sqrt{-\lambda_{1}\left(\theta_{0}\right)} \int_{\Omega}\left|\tilde{u}_{\epsilon}(T)\right|^{2} d x+\int_{0}^{T} \int_{\Omega}\left(A^{\epsilon} \nabla \tilde{u}_{\epsilon} \cdot \nabla \tilde{u}_{\epsilon}+\frac{c^{\epsilon}-\lambda_{1}\left(\theta_{0}\right)}{\epsilon^{2}} \tilde{u}_{\epsilon} \cdot \tilde{u}_{\epsilon}\right) d x d t= \\
& \sqrt{-\lambda_{1}\left(\theta_{0}\right)} \int_{\Omega}\left|\tilde{u}_{\epsilon}(0)\right|^{2} d x+\epsilon^{2} \int_{0}^{T} \int_{\Omega}\left|\frac{\partial \tilde{u}_{\epsilon}}{\partial t}\right|^{2} d x d t \\
& +\epsilon^{2} \int_{\Omega} \tilde{u}_{\epsilon}(0) \frac{\partial \tilde{u}_{\epsilon}}{\partial t}(0) d x-\epsilon^{2} \int_{\Omega} \tilde{u}_{\epsilon}(T) \frac{\partial \tilde{u}_{\epsilon}}{\partial t}(T) d x .
\end{aligned}
$$

Using 76 we deduce from 77 )

$$
\sqrt{-\lambda_{1}\left(\theta_{0}\right)}\left\|\tilde{u}_{\epsilon}(T)\right\|_{L^{2}(\Omega)^{K}}^{2} \leq C\left(1+\left\|\tilde{u}_{\epsilon}(T)\right\|_{L^{2}(\Omega)^{K}}\right),
$$

which implies that $\tilde{u}_{\epsilon}$ is bounded in $L^{\infty}\left((0, T) ; L^{2}(\Omega)^{K}\right)$. Using this information in (77) shows that assumption (55) is satisfied: thus, Lemma 4.2 can be applied to obtain (73) and (74).

Finally we arrive at the proof of Theorem 3.10 when $\lambda_{1}\left(\theta_{0}\right)>0$ and again we simply address the question of uniform a priori estimates for (41) (the proof of convergence is an adaptation of Theorem 3.2 and of the arguments of [3] in the scalar case).

Lemma 6.3 Under the assumptions of Theorem 3.10 the solution $\tilde{u}_{\epsilon}$ of (41) satisfies

$$
\left\|\tilde{u}_{\epsilon}\right\|_{L^{\infty}\left((0, T) ; L^{2}(\Omega)^{K}\right)}+\epsilon\left\|\nabla \tilde{u}_{\epsilon}\right\|_{L^{2}((0, T) \times \Omega)^{N \times K}}+\epsilon\left\|\frac{\partial \tilde{u}_{\epsilon}}{\partial t}\right\|_{L^{2}((0, T) \times \Omega)^{K}} \leq C,
$$

where $C>0$ is a constant which does not depend on $\epsilon$.

Remark 6.4 The a priori estimates of Lemma 6.3 are weaker than the previous ones and in particular do not allow us to recover the homogenized Dirichlet boundary condition. As in the scalar case [3], in order to obtain the homogenized boundary condition the trick is to study the homogenization of a time integral of (41) which has less oscillating initial data. Indeed, defining $w_{\epsilon}(t, x)=\int_{0}^{t} \tilde{u}_{\epsilon}(s, x) d s+\chi_{\epsilon}(x)$ with a suitable choice of $\chi_{\epsilon}$ (so that $w_{\epsilon}$ satisfies the same p.d.e. than (41) without source term), one can obtain better a priori estimates for $w_{\epsilon}$ than for $\tilde{u}_{\epsilon}$. 
Proof. In a first step we multiply 41 by $\frac{\partial \overline{\tilde{u}_{\epsilon}}}{\partial t}$ and we take the real part to obtain the usual energy estimate

$$
E_{\epsilon}(t)=E_{\epsilon}(0)
$$

with $E_{\epsilon}(t)=\frac{1}{2} \int_{\Omega}\left(\epsilon^{2}\left|\frac{\partial \tilde{u}_{\epsilon}}{\partial t}\right|^{2}+A^{\epsilon} \nabla \tilde{u}_{\epsilon} \cdot \nabla \overline{\tilde{u}_{\epsilon}}+\frac{c^{\epsilon}-\lambda_{1}\left(\theta_{0}\right)}{\epsilon^{2}} \tilde{u}_{\epsilon} \cdot \overline{\tilde{u}}_{\epsilon}\right) d x$. As in the proof of Lemma 6.2 , the assumptions merely imply

$$
E_{\epsilon}(0) \leq C \epsilon^{-2}
$$

Nevertheless, from the Bloch wave analysis of Lemma 3.1 we deduce

$$
\int_{\Omega}\left(A^{\epsilon} \nabla \tilde{u}_{\epsilon} \cdot \nabla \tilde{\tilde{u}}_{\epsilon}+\frac{c^{\epsilon}-\lambda_{1}\left(\theta_{0}\right)}{\epsilon^{2}} \tilde{u}_{\epsilon} \cdot \overline{\tilde{u}}_{\epsilon}\right) d x \geq 0
$$

which, combined with (79), yields

$$
\epsilon^{2}\left\|\frac{\partial \tilde{u}_{\epsilon}}{\partial t}\right\|_{L^{\infty}\left((0, T) ; L^{2}(\Omega)^{K}\right)} \leq C .
$$

In a second step we multiply 41 by $\bar{u}_{\epsilon}$ and we take the imaginary part

$$
\begin{aligned}
& \sqrt{\lambda_{1}\left(\theta_{0}\right)} \int_{\Omega}\left|\tilde{u}_{\epsilon}(T)\right|^{2} d x-\sqrt{\lambda_{1}\left(\theta_{0}\right)} \int_{\Omega}\left|\tilde{u}_{\epsilon}(0)\right|^{2} d x \\
& +\epsilon^{2} \mathcal{I}\left(\int_{\Omega} \tilde{\tilde{u}}_{\epsilon}(T) \frac{\partial \tilde{u}_{\epsilon}}{\partial t}(T) d x-\int_{\Omega} \tilde{\tilde{u}}_{\epsilon}(0) \frac{\partial \tilde{u}_{\epsilon}}{\partial t}(0) d x\right)=0 .
\end{aligned}
$$

Using 80 we deduce from 81

$$
\sqrt{\lambda_{1}\left(\theta_{0}\right)}\left\|\tilde{u}_{\epsilon}(T)\right\|_{L^{2}(\Omega)^{K}}^{2} \leq C\left(1+\left\|\tilde{u}_{\epsilon}(T)\right\|_{L^{2}(\Omega)^{K}}\right)
$$

which implies that $\tilde{u}_{\epsilon}$ is bounded in $L^{\infty}\left((0, T) ; L^{2}(\Omega)^{K}\right)$. Remark that 81$)$, unlike (77), does not include any gradient term, so we can not apply Lemma 4.2 to obtain a better estimate.

\section{Generalization to high level bands}

We generalize the homogenization of a parabolic system established in Section 3 for initial data concentrating at the bottom of the first Bloch band to 
another type of initial data concentrating at the bottom of an higher level band. Such a generalization holds true only in the case of the whole space $\Omega=\mathbb{R}^{N}$ because otherwise we lack an adequate generalization of the compactness Lemma 4.2, ¿From now on in this section we replace assumption (9) by the following one: for an energy level $n \geq 1$, there exists a Bloch parameter $\theta_{0} \in \mathbb{T}^{N}$ such that

$$
\begin{cases}(i) & \theta_{0} \text { is the unique minimizer of } \lambda_{n}(\theta) \text { in } \mathbb{T}^{N} \\ (\text { ii }) & \lambda_{n}\left(\theta_{0}\right) \text { is a simple eigenvalue, } \\ (\text { iii }) & \text { the Hessian matrix } \nabla_{\theta} \nabla_{\theta} \lambda_{n}\left(\theta_{0}\right) \text { is positive definite. }\end{cases}
$$

Under assumption (82) the $n^{\text {th }}$ eigencouple of $(8)$ is analytic at $\theta_{0}$. It is easily seen that the first derivative $\frac{\partial \psi_{n}}{\partial \theta_{k}}$ and the second derivative $\frac{\partial^{2} \psi_{n}}{\partial \theta_{k} \partial \theta_{l}}$ satisfy equations similar to (11) and (12) respectively, up to changing the label 1 in $n$. In particular, for $\theta=\theta_{0}$ we still use the following notation

$$
\frac{\partial \psi_{n}}{\partial \theta_{k}}=2 i \pi \zeta_{k}, \quad \frac{\partial^{2} \psi_{n}}{\partial \theta_{k} \partial \theta_{l}}=-4 \pi^{2} \chi_{k l}
$$

where $\zeta_{k}$ and $\chi_{k l}$ are solutions of (14) and (15) respectively, up to changing the label 1 in $n$.

We study a parabolic system with purely periodic coefficients

$$
\begin{cases}\frac{\partial u_{\epsilon}}{\partial t}-\operatorname{div}\left(A\left(\frac{x}{\epsilon}\right) \nabla u_{\epsilon}\right)+\frac{c\left(\frac{x}{\epsilon}\right)}{\epsilon^{2}} u_{\epsilon}=0 & \text { in } \mathbb{R}^{N} \times(0, T) \\ u_{\epsilon}(t=0, x)=u_{\epsilon}^{0}(x) & \text { in } \mathbb{R}^{N}\end{cases}
$$

We also need an assumption on the initial data which must be "well prepared", namely concentrating at the bottom on the $n^{\text {th }}$ Bloch band. Recall from Lemma 2.4 that any function $u_{\epsilon}^{0} \in L^{2}\left(\mathbb{R}^{N}\right)$ can be decomposed as

$$
u_{\epsilon}^{0}(x)=\sum_{k \geq 1} \int_{\epsilon^{-1} \mathbb{T}^{N}} \alpha_{k}^{\epsilon}(\eta) \psi_{k}\left(\frac{x}{\epsilon}, \theta_{0}+\epsilon \eta\right) e^{2 i \pi \eta \cdot x} e^{-2 i \pi \frac{\theta_{0} \cdot x}{\epsilon}} d \eta,
$$

with $\eta=\frac{\theta-\theta_{0}}{\epsilon}$. We denote by $\Pi_{\epsilon}^{n}$ the projection operator on the Bloch bands above the $n^{t h}$ level

$$
\Pi_{\epsilon}^{n} u_{\epsilon}^{0}(x)=\sum_{k \geq n} \int_{\epsilon^{-1} \mathbb{T}^{N}} \alpha_{k}^{\epsilon}(\eta) \psi_{k}\left(\frac{x}{\epsilon}, \theta_{0}+\epsilon \eta\right) e^{2 i \pi \eta \cdot x} e^{-2 i \pi \frac{\theta_{0} \cdot x}{\epsilon}} d \eta
$$


Our assumption on the initial data is that

$$
u_{\epsilon}^{0}=\Pi_{\epsilon}^{n} u_{\epsilon}^{0}
$$

Typically, we are interested in an initial data of the type

$$
u_{\epsilon}^{0,1}(x)=\Pi_{\epsilon}^{n}\left(v^{0}(x) \psi_{n}\left(\frac{x}{\epsilon}, \theta_{0}\right) e^{2 i \pi \frac{\theta_{0} \cdot x}{\epsilon}}\right),
$$

with $v^{0} \in L^{2}\left(\mathbb{R}^{N}\right)$. However, since the projection operator $\Pi_{\epsilon}^{n}$ is not very explicit, we also consider another type of initial data which satisfies assumption (86), namely

$$
u_{\epsilon}^{0,2}(x)=\int_{\epsilon^{-1} \mathbb{T}^{N}} \alpha_{n}(\eta) \psi_{n}\left(\frac{x}{\epsilon}, \theta_{0}+\epsilon \eta\right) e^{2 i \pi \eta \cdot x} e^{2 i \pi \frac{\theta_{0} \cdot x}{\epsilon}} d \eta
$$

with $\alpha_{n} \in L^{2}\left(\mathbb{R}^{N}\right)$ being the Fourier transform of $v^{0}(x)$. Actually, it is easy to check that

$$
\lim _{\epsilon \rightarrow 0}\left\|u_{\epsilon}^{0,1}-u_{\epsilon}^{0,2}\right\|_{L^{2}\left(\mathbb{R}^{N}\right)}=0 .
$$

For such well-prepared initial data, we perform a time renormalization similar to 18

$$
\tilde{u}_{\epsilon}(t, x)=e^{\frac{\lambda_{n}\left(\theta_{0}\right) t}{\epsilon^{2}}} u_{\epsilon}(t, x)
$$

such that $\tilde{u}_{\epsilon}$ satisfies

$$
\begin{cases}\frac{\partial \tilde{u}_{\epsilon}}{\partial t}-\operatorname{div}\left(A\left(\frac{x}{\epsilon}\right) \nabla \tilde{u}_{\epsilon}\right)+\frac{c\left(\frac{x}{\epsilon}\right)-\lambda_{n}\left(\theta_{0}\right)}{\epsilon^{2}} \tilde{u}_{\epsilon}=0 & \text { in } \mathbb{R}^{N} \times(0, T), \\ \tilde{u}_{\epsilon}(t=0, x)=u_{\epsilon}^{0}(x) & \text { in } \mathbb{R}^{N}\end{cases}
$$

Lemma 7.1 Under assumption (86), the solution of (90) satisfies

$$
\left\|\tilde{u}_{\epsilon}\right\|_{L^{\infty}\left((0, T) ; L^{2}\left(\mathbb{R}^{N}\right)^{K}\right)}+\epsilon\left\|\nabla \tilde{u}_{\epsilon}\right\|_{L^{2}\left((0, T) \times \mathbb{R}^{N}\right)^{N \times K}} \leq C\left\|u_{\epsilon}^{0}\right\|_{L^{2}\left(\mathbb{R}^{N}\right)^{K}}
$$

and there exists a bounded scalar sequence $v_{\epsilon}$ in $L^{2}\left((0, T) ; H^{1}\left(\mathbb{R}^{N}\right)\right)$ such that

$$
\tilde{u}_{\epsilon}(t, x)=\psi_{n}\left(\frac{x}{\epsilon}, \theta_{0}\right) e^{2 i \pi \frac{\theta_{0} \cdot x}{\epsilon}} v_{\epsilon}(t, x)+r_{\epsilon}(t, x),
$$

where $\lim _{\epsilon \rightarrow 0}\left\|r_{\epsilon}\right\|_{L^{2}((0, T) \times \omega)^{K}}=0$ for any compact set $\omega \subset \mathbb{R}^{N}$. 
Theorem 7.2 Assume that the initial data $u_{\epsilon}^{0} \in L^{2}\left(\mathbb{R}^{N}\right)^{K}$ is of the form (87) or (88). The solution of (84) can be written as

$$
u_{\epsilon}(t, x)=e^{-\frac{\lambda_{n}\left(\theta_{0}\right) t}{\epsilon^{2}}}\left(\psi_{n}\left(\frac{x}{\epsilon}, \theta_{0}\right) e^{2 i \pi \frac{\theta_{0} \cdot x}{\epsilon}} v_{\epsilon}(t, x)+r_{\epsilon}(t, x)\right),
$$

where $r_{\epsilon}$ is a remainder term such that

$$
\lim _{\epsilon \rightarrow 0}\left\|r_{\epsilon}\right\|_{L^{2}((0, T) \times \omega)^{K}}=0 \text { for any compact set } \omega \subset \mathbb{R}^{N},
$$

and $v_{\epsilon}$ converges weakly in $L^{2}\left((0, T) ; H^{1}\left(\mathbb{R}^{N}\right)\right)$ to the solution $v$ of the scalar homogenized problem

$$
\begin{cases}\frac{\partial v}{\partial t}-\operatorname{div}\left(A_{n}^{*} \nabla v\right)=0 & \text { in } \mathbb{R}^{N} \times(0, T), \\ v(t=0, x)=v^{0}(x) & \text { in } \mathbb{R}^{N}\end{cases}
$$

with $A_{n}^{*}=\frac{1}{4 \pi^{2}} \nabla_{\theta} \nabla_{\theta} \lambda_{n}\left(\theta_{0}\right)$.

Remark 7.3 In the context of the Schrödinger equation Theorem 7.2 is called an effective mass theorem [22], [24], [25]. Even in the case of a scalar equation, Theorem 7.2 is new since the factorization principle does not work for an energy level $n>1$, namely one can not divide the unknown $u_{\epsilon}$ by $\psi_{n}\left(\frac{x}{\epsilon}, \theta_{0}\right)$ which necessarily vanishes at some points in $\mathbb{T}^{N}$.

Remark 7.4 An initial data of the type (87) or (88) would yield a zero limit if homogenized in the setting of Theorem 3.2. The solution $u_{\epsilon}$, given by (93), decays much faster than that given by 22) because $\lambda_{n}\left(\theta_{0}\right)>\lambda_{1}\left(\theta_{0}\right)$. Therefore, we can interpret Theorem 7.2 as describing initial layers in time, compared to Theorem 3.2 which captures the average behavior. This is consistent with the classical homogenization of parabolic equations, when $c \equiv 0$, where initial layers in time are known to exist [12] but can not be characterized by the classical homogenization theory.

Proof of Lemma 7.1. We apply the rescaled Bloch decomposition (16) to equation 90

$$
\tilde{u}_{\epsilon}(t, x)=\sum_{k \geq 1} \int_{\epsilon^{-1} \mathbb{T}^{N}} \alpha_{k}^{\epsilon}(t, \eta) \psi_{k}\left(\frac{x}{\epsilon}, \theta_{0}+\epsilon \eta\right) e^{2 i \pi \eta \cdot x} e^{2 i \pi \frac{\theta_{0} \cdot x}{\epsilon}} d \eta
$$


with

$$
\alpha_{k}^{\epsilon}(t, \eta)=\alpha_{k}^{\epsilon}(0, \eta) e^{\frac{\left(\lambda_{n}-\lambda_{k}\right)\left(\theta_{0}+\epsilon \eta\right)}{\epsilon^{2}} t} .
$$

¿From assumption 86 we deduce that $\alpha_{k}^{\epsilon}(t, \eta)=0$ for any $k<n$. Therefore, for any time $t$, we have $\Pi_{\epsilon}^{n} \tilde{u}_{\epsilon}(t, x)=\tilde{u}_{\epsilon}(t, x)$. Thus,

$$
\int_{\mathbb{R}^{N}}\left(A\left(\frac{x}{\epsilon}\right) \nabla \tilde{u}_{\epsilon} \cdot \nabla \tilde{u}_{\epsilon}+\frac{c\left(\frac{x}{\epsilon}\right)-\lambda_{n}\left(\theta_{0}\right)}{\epsilon^{2}} \tilde{u}_{\epsilon} \cdot \tilde{u}_{\epsilon}\right) d x \geq 0
$$

which easily yields the a priori estimate (91). We now mimick the arguments of the proof of Lemma 4.2 (replacing the label 1 by $n$ ) to obtain the compactness result $(92)$.

Proof of Theorem 7.2. The proof is very similar to that of Theorem 3.2 so we simply sketch the main points. We introduce, as before, a sequence $w_{\epsilon}$ defined by

$$
w_{\epsilon}(t, x)=\tilde{u}_{\epsilon}(t, x) e^{-2 i \pi \frac{\theta_{0} \cdot x}{\epsilon}} .
$$

By the a priori estimates of Lemma 7.1, there exist a subsequence and a limit $w(t, x, y) \in L^{2}\left((0, T) \times \mathbb{R}^{N} ; H^{1}\left(\mathbb{T}^{N}\right)^{K}\right)$ such that $w_{\epsilon}$ and $\epsilon \nabla w_{\epsilon}$ twoscale converges to $w$ and $\nabla_{y} w$ respectively [2], [23]). Similarly, by its very definition, $w_{\epsilon}(0, x)$ two-scale converges to $\psi_{n}\left(y, \theta_{0}\right) v^{0}(x)$. In a first step we multiply 90 by the complex conjugate of $\epsilon^{2} \phi\left(t, x, \frac{x}{\epsilon}\right) e^{2 i \pi \frac{\theta_{0} \cdot x}{\epsilon}}$ where $\phi(t, x, y)$ is a smooth test function defined in $[0, T) \times \mathbb{R}^{N} \times \mathbb{T}^{N}$ with values in $\mathbb{C}^{K}$. Passing to the two-scale limit yields the existence of a scalar function $v(t, x) \in$ $L^{2}\left((0, T) \times \mathbb{R}^{N}\right)$ such that $w(t, x, y)=v(t, x) \psi_{n}\left(y, \theta_{0}\right)$. In a second step we multiply $(90)$ by the complex conjugate of

$$
\Psi_{\epsilon}=e^{2 i \pi \frac{\theta_{0} \cdot x}{\epsilon}}\left(\psi_{n}\left(\frac{x}{\epsilon}, \theta_{0}\right) \phi(t, x)+\epsilon \sum_{k=1}^{N} \frac{\partial \phi}{\partial x_{k}}(t, x) \zeta_{k}\left(\frac{x}{\epsilon}\right)\right)
$$

where $\phi(t, x)$ is a smooth, compactly supported, test function defined from $[0, T) \times \mathbb{R}^{N}$ into $\mathbb{R}$, and $\zeta_{k}(y)$ is the solution of $(14)$ where the label 1 is replaced by $n$. Passing to the two-scale limit yields a very weak form of the homogenized equation (95). It is routine to show that its solution $v(t, x)$ is indeed a classical weak solution. Then, by uniqueness of the solution, we deduce that the entire sequence $w_{\epsilon}$ two-scale converges to $\psi_{n}\left(y, \theta_{0}\right) v(t, x)$. 
Remark 7.5 All the results of this section are specific to the case of the whole space, i.e. $\Omega=\mathbb{R}^{N}$, and can not be extended to the case of an additional zero-order term $d\left(x, \frac{x}{\epsilon}\right)$ because we crucially use the Bloch diagonalization to get a priori estimates.

\section{Fourth order homogenized problem}

By changing the main assumption on the Bloch spectrum it is possible to obtain a fourth order homogenized equation from a second order parabolic problem. Specifically we consider

$$
\begin{cases}\epsilon^{2} \frac{\partial u_{\epsilon}}{\partial t}-\operatorname{div}\left(A\left(\frac{x}{\epsilon}\right) \nabla u_{\epsilon}\right)+\left(\epsilon^{-2} c\left(\frac{x}{\epsilon}\right)+\epsilon^{2} d\left(x, \frac{x}{\epsilon}\right)\right) u_{\epsilon}=0 & \text { in } \Omega \times(0, T), \\ u_{\epsilon}=0 & \text { on } \partial \Omega \times(0, T), \\ u_{\epsilon}(t=0, x)=u_{\epsilon}^{0}(x) & \text { in } \Omega .\end{cases}
$$

Remark that the time scaling in $(96)$ is not the same than that in (17): this means that we are looking for an asymptotic for longer time of order $\epsilon^{-2}$ in (96), compared to (17). Instead of (9), we now make the following assumption

$$
\left\{\begin{array}{l}
(i) \quad \theta_{0} \text { is the unique minimizer of } \lambda_{1}(\theta) \text { in } \mathbb{T}^{N} \\
(\text { ii }) \quad \lambda_{1}\left(\theta_{0}\right) \text { is a simple eigenvalue, } \\
\text { (iii) } \nabla_{\theta} \nabla_{\theta} \lambda_{1}\left(\theta_{0}\right)=0, \\
\text { (iv) the fourth-order tensor } \nabla_{\theta} \nabla_{\theta} \nabla_{\theta} \nabla_{\theta} \lambda_{1}\left(\theta_{0}\right) \text { is positive definite. }
\end{array}\right.
$$

Remark 8.1 We do not know if assumption (97) is satisfied for any practical example.

Since $\lambda_{1}\left(\theta_{0}\right)$ is a minimum, we also have $\nabla_{\theta} \lambda_{1}\left(\theta_{0}\right)=0$ and $\nabla_{\theta} \nabla_{\theta} \nabla_{\theta} \lambda_{1}\left(\theta_{0}\right)=$ 0 . Under assumption (97) the first eigencouple of (8) is analytic at $\theta_{0}$. Recall that, for $\theta=\theta_{0}$, the two first derivatives of $\psi_{1}$ are given by

$$
\frac{\partial \psi_{1}}{\partial \theta_{k}}=2 i \pi \zeta_{k}, \quad \frac{\partial^{2} \psi_{1}}{\partial \theta_{k} \partial \theta_{l}}=-4 \pi^{2} \chi_{k l},
$$

where $\zeta_{k}$ is the solution of (14) and $\chi_{k l}$ is the solution of (15) (remark that this last equation simplifies since $\left.\nabla_{\theta} \nabla_{\theta} \lambda_{1}\left(\theta_{0}\right)=0\right)$. Similarly, the third 
derivative is

$$
\frac{\partial^{3} \psi_{1}}{\partial \theta_{j} \partial \theta_{k} \partial \theta_{l}}=-8 i \pi^{3} \xi_{j k l},
$$

where

$$
\begin{aligned}
\mathbb{A}\left(\theta_{0}\right) \xi_{j k l} & =e_{j} A(y)\left(\nabla_{y}+2 i \pi \theta_{0}\right) \chi_{k l}+\left(\operatorname{div}_{y}+2 i \pi \theta_{0}\right)\left(A(y) e_{j} \chi_{k l}\right) \\
& +e_{k} A(y)\left(\nabla_{y}+2 i \pi \theta_{0}\right) \chi_{j l}+\left(\operatorname{div}_{y}+2 i \pi \theta_{0}\right)\left(A(y) e_{k} \chi_{j l}\right) \\
& +e_{l} A(y)\left(\nabla_{y}+2 i \pi \theta_{0}\right) \chi_{k j}+\left(\operatorname{div}_{y}+2 i \pi \theta_{0}\right)\left(A(y) e_{l} \chi_{k j}\right) \\
& +e_{k} A(y) e_{l} \zeta_{j}+e_{j} A(y) e_{l} \zeta_{k}+e_{k} A(y) e_{j} \zeta_{l} .
\end{aligned}
$$

There exists a unique solution of (100), up to the addition of a multiple of $\psi_{1}$. Indeed, the right hand side of (100) satisfies the required compatibility condition (i.e. it is orthogonal to $\psi_{1}$ ) because all derivatives of $\lambda_{1}(\theta)$, up to third order, are zero at $\theta=\theta_{0}$.

We perform a time renormalization by introducing a new unknown

$$
\tilde{u}_{\epsilon}(t, x)=e^{\frac{\lambda_{1}\left(\theta_{0}\right) t}{\epsilon^{4}}} u_{\epsilon}(t, x)
$$

which satisfies

$$
\begin{cases}\frac{\partial \tilde{u}_{\epsilon}}{\partial t}-\epsilon^{-2} \operatorname{div}\left(A\left(\frac{x}{\epsilon}\right) \nabla \tilde{u}_{\epsilon}\right)+\frac{c\left(\frac{x}{\epsilon}\right)-\lambda_{1}\left(\theta_{0}\right)}{\epsilon^{4}} \tilde{u}_{\epsilon}+d\left(x, \frac{x}{\epsilon}\right) \tilde{u}_{\epsilon}=0 & \text { in } \Omega \times(0, T), \\ \tilde{u}_{\epsilon}=0 & \text { on } \partial \Omega \times(0, T), \\ \tilde{u}_{\epsilon}(t=0, x)=u_{\epsilon}^{0}(x) & \text { in } \Omega .\end{cases}
$$

As usual we obtain the following a priori estimate

$$
\left\|\tilde{u}_{\epsilon}\right\|_{L^{\infty}\left((0, T) ; L^{2}(\Omega)^{K}\right)}+\epsilon\left\|\nabla \tilde{u}_{\epsilon}\right\|_{L^{2}((0, T) \times \Omega)^{N \times K}} \leq C\left\|u_{\epsilon}^{0}\right\|_{L^{2}(\Omega)^{K}}
$$

where the constant $C>0$ does not depend on $\epsilon$.

Theorem 8.2 Assume that the initial data $u_{\epsilon}^{0} \in L^{2}(\Omega)^{K}$ is of the form

$$
u_{\epsilon}^{0}(x)=\psi_{1}\left(\frac{x}{\epsilon}, \theta_{0}\right) e^{2 i \pi \frac{\theta_{0} \cdot x}{\epsilon}} v^{0}(x)
$$

with $v^{0} \in L^{2}(\Omega)$. The solution of 96 can be written as

$$
u_{\epsilon}(t, x)=e^{-\frac{\lambda_{1}\left(\theta_{0}\right) t}{\epsilon^{4}}}\left(\psi_{1}\left(\frac{x}{\epsilon}, \theta_{0}\right) e^{2 i \pi \frac{\theta_{0} \cdot x}{\epsilon}} v_{\epsilon}(t, x)+r_{\epsilon}(t, x)\right),
$$


where $r_{\epsilon}$ is a remainder term such that

$$
\lim _{\epsilon \rightarrow 0}\left\|r_{\epsilon}\right\|_{L^{2}((0, T) \times \omega)^{K}}=0 \text { for any compact set } \omega \subset \mathbb{R}^{N},
$$

and $v_{\epsilon}$ converges weakly in $L^{2}\left((0, T) ; H^{2}(\Omega)\right)$ to the solution $v$ of the scalar fourth-order homogenized problem

$$
\begin{cases}\frac{\partial v}{\partial t}+\operatorname{div} \operatorname{div}\left(A^{*} \nabla \nabla v\right)=0 & \text { in } \Omega \times(0, T), \\ \frac{\partial v}{\partial n}=v=0 & \text { on } \partial \Omega \times(0, T), \\ v(t=0, x)=v^{0}(x) & \text { in } \Omega,\end{cases}
$$

with $A^{*}=\frac{1}{16 \pi^{4}} \nabla_{\theta} \nabla_{\theta} \nabla_{\theta} \nabla_{\theta} \lambda_{1}\left(\theta_{0}\right)$.

To prove Theorem 8.2 we need the following generalization of Lemma 4.2 . Lemma 8.3 Let $u_{\epsilon}$ be a bounded sequence in $L^{2}\left(\mathbb{R}^{N}\right)^{K}$. Assume that there exists a finite constant $C$ such that

$$
\int_{\mathbb{R}^{N}}\left(A\left(\frac{x}{\epsilon}\right) \nabla u_{\epsilon} \cdot \nabla u_{\epsilon}+\frac{c\left(\frac{x}{\epsilon}\right)-\lambda_{1}\left(\theta_{0}\right)}{\epsilon^{2}} u_{\epsilon} \cdot u_{\epsilon}\right) d x \leq C \epsilon^{2} .
$$

Then, under assumption (97),

$$
u_{\epsilon}(x)=\psi_{1}\left(\frac{x}{\epsilon}, \theta_{0}\right) e^{2 i \pi \frac{\theta_{0} \cdot x}{\epsilon}} v_{\epsilon}(x)+r_{\epsilon}(x),
$$

where $v_{\epsilon}$ is a bounded scalar sequence in $H^{2}\left(\mathbb{R}^{N}\right)$ and $\lim _{\epsilon \rightarrow 0}\left\|r_{\epsilon}\right\|_{L^{2}(\omega)^{K}}=0$ for any compact set $\omega \subset \mathbb{R}^{N}$.

Proof. Introducing the rescaled Bloch decomposition (16) of $u_{\epsilon}(x)$ with $\eta=\frac{\theta-\theta_{0}}{\epsilon}$,

$$
u_{\epsilon}(x)=\sum_{k \geq 1} \int_{\epsilon^{-1} \mathbb{T}^{N}} \alpha_{k}^{\epsilon}(\eta) \psi_{k}\left(\frac{x}{\epsilon}, \theta_{0}+\epsilon \eta\right) e^{2 i \pi \eta \cdot x} e^{2 i \pi \frac{\theta_{0} \cdot x}{\epsilon}} d \eta
$$

the same arguments than those in the proof of Lemma 4.2 and the estimate

$$
\lambda_{1}(\theta)-\lambda_{1}\left(\theta_{0}\right) \geq C\left|\theta-\theta_{0}\right|^{4} \quad \forall \theta \in \mathbb{T}^{N},
$$


shows that

$$
\int_{\epsilon^{-1} \mathbb{T}^{N}}|\eta|^{4}\left|\alpha_{1}^{\epsilon}(\eta)\right|^{2} d \eta \leq C
$$

Defining $v_{\epsilon}(x)$ as the inverse Fourier transform of $\alpha_{1}^{\epsilon}(\eta)$, we deduce that $v_{\epsilon}$ is uniformly bounded in $H^{2}\left(\mathbb{R}^{N}\right)$.

Proof of Theorem 8.2, The proof is similar to that of Theorem 3.2 . The first step is identical: the function $w_{\epsilon}(t, x)=u_{\epsilon}(t, x) e^{-2 i \pi \frac{\theta_{0} \cdot x}{\epsilon}}$ two-scale converges to a limit $v(t, x) \psi_{1}\left(y, \theta_{0}\right)$. In the second step, we multiply (102) by the complex conjugate of

$$
\begin{aligned}
\Psi_{\epsilon}= & e^{2 i \pi \frac{\theta_{0} \cdot x}{\epsilon}}\left(\psi_{1}\left(\frac{x}{\epsilon}, \theta_{0}\right) \phi(t, x)+\epsilon \sum_{k=1}^{N} \frac{\partial \phi}{\partial x_{k}}(t, x) \zeta_{k}\left(\frac{x}{\epsilon}\right)\right. \\
& \left.+\epsilon^{2} \sum_{k, l=1}^{N} \frac{\partial^{2} \phi}{\partial x_{k} \partial x_{l}}(t, x) \chi_{k l}\left(\frac{x}{\epsilon}\right)+\epsilon^{3} \sum_{j, k, l=1}^{N} \frac{\partial^{3} \phi}{\partial x_{j} \partial x_{k} \partial x_{l}}(t, x) \xi_{j k l}\left(\frac{x}{\epsilon}\right)\right),
\end{aligned}
$$

where $\phi(t, x)$ is a smooth, compactly supported, test function defined from $[0, T) \times \Omega$ into $\mathbb{R}, \zeta_{k}(y)$ is the solution of $(14), \chi_{k l}(y)$ is the solution of $(15)$, and $\xi_{j k l}(y)$ is the solution of 100 . After some tedious algebra we find that

$$
\begin{aligned}
& \int_{\Omega}\left|\psi_{1}^{\epsilon}\right|^{2} v^{0} \bar{\phi}(0) d x-\int_{0}^{T} \int_{\Omega} w_{\epsilon} \cdot \bar{\psi}_{1}^{\epsilon} \frac{\partial \bar{\phi}}{\partial t} d t d x \\
& -\int_{0}^{T} \int_{\Omega} A^{\epsilon} w_{\epsilon} \nabla \frac{\partial^{3} \bar{\phi}}{\partial x_{j} \partial x_{k} \partial x_{l}} \cdot e_{k} \bar{\chi}_{j l}^{\epsilon} d t d x \\
& -\int_{0}^{T} \int_{\Omega} A^{\epsilon} w_{\epsilon} \nabla \frac{\partial^{3} \bar{\phi}}{\partial x_{j} \partial x_{k} \partial x_{l}} \cdot\left(\epsilon \nabla-2 i \pi \theta_{0}\right) \bar{\eta}_{j k l}^{\epsilon} d t d x \\
& +\int_{0}^{T} \int_{\Omega} A^{\epsilon} \bar{\eta}_{j k l}^{\epsilon}\left(\epsilon \nabla+2 i \pi \theta_{0}\right) w_{\epsilon} \cdot \nabla \frac{\partial^{3} \bar{\phi}}{\partial x_{j} \partial x_{k} \partial x_{l}} d t d x \\
& +\int_{0}^{T} \int_{\Omega} d^{\epsilon} w_{\epsilon} \cdot \bar{\psi}_{1}^{\epsilon} \bar{\phi} d t d x
\end{aligned}
$$


Passing to the two-scale limit in each term of 109 gives

$$
\begin{aligned}
& \int_{\Omega} \int_{\mathbb{T}^{N}}\left|\psi_{1}\right|^{2} v^{0} \bar{\phi}(0) d x d y-\int_{0}^{T} \int_{\Omega} \int_{\mathbb{T}^{N}}\left|\psi_{1}\right|^{2} v \frac{\partial \bar{\phi}}{\partial t} d t d x d y \\
& -\int_{0}^{T} \int_{\Omega} \int_{\mathbb{T}^{N}} A \psi_{1} v \nabla \frac{\partial^{3} \bar{\phi}}{\partial x_{j} \partial x_{k} \partial x_{l}} \cdot e_{k} \bar{\chi}_{j l} d t d x d y \\
& -\int_{0}^{T} \int_{\Omega} \int_{\mathbb{T}^{N}} A \psi_{1} v \nabla \frac{\partial^{3} \bar{\phi}}{\partial x_{j} \partial x_{k} \partial x_{l}} \cdot\left(\nabla_{y}-2 i \pi \theta_{0}\right) \bar{\eta}_{j k l} d t d x d y \\
& +\int_{0}^{T} \int_{\Omega} \int_{\mathbb{T}^{N}} A \bar{\eta}_{j k l}\left(\nabla_{y}+2 i \pi \theta_{0}\right) \psi_{1} v \cdot \nabla \frac{\partial^{3} \bar{\phi}}{\partial x_{j} \partial x_{k} \partial x_{l}} d t d x d y \\
& +\int_{0}^{T} \int_{\Omega} \int_{\mathbb{T}^{N}} d \psi_{1} v \cdot \bar{\psi}_{1} \bar{\phi} d t d x d y
\end{aligned}
$$

Recalling the normalization $\int_{\mathbb{T}^{N}}\left|\psi_{1}\right|^{2} d y=1$, and introducing

$$
\begin{aligned}
A_{j k l m}^{*}=\int_{\mathbb{T}^{N}} & \left(-A \psi_{1} e_{m} \cdot e_{k} \bar{\chi}_{j l}-A \psi_{1} e_{m} \cdot\left(\nabla_{y}-2 i \pi \theta_{0}\right) \bar{\eta}_{j k l}\right. \\
& \left.+A \bar{\eta}_{j k l}\left(\nabla_{y}+2 i \pi \theta_{0}\right) \psi_{1} \cdot e_{m}\right) d y
\end{aligned}
$$

(which has to be symmetrized), and $d^{*}(x)=\int_{\mathbb{T}^{N}} d(x, y) \psi_{1}(y) \cdot \bar{\psi}_{1}(y) d y, 110$ is equivalent to

$$
\int_{\Omega} v^{0} \bar{\phi}(0) d x-\int_{0}^{T} \int_{\Omega}\left(v \frac{\partial \bar{\phi}}{\partial t}-A^{*} v \cdot \nabla \nabla \nabla \nabla \bar{\phi}-d^{*}(x) v \bar{\phi}\right) d t d x=0
$$

which is a very weak form of the homogenized equation (105). To recover the Dirichlet boundary condition, we use Lemma 8.3 which implies that $v \in H^{2}\left(\mathbb{R}^{N}\right)$ and $v=0$ in any compact set $\omega \subset\left(\mathbb{R}^{N} \backslash \Omega\right)$. Thus $v$ belongs to $H_{0}^{2}(\Omega)$.

The compatibility condition of the equation giving the fourth derivative of $\psi_{1}$ yields that the tensor $A^{*}$, defined by (111), is indeed equal to $\frac{1}{16 \pi^{4}} \nabla_{\theta} \nabla_{\theta} \nabla_{\theta} \nabla_{\theta} \lambda_{1}\left(\theta_{0}\right)$, and thus is real, symmetric, positive definite by assumption (97). Therefore, the homogenized problem (105) is well posed. By uniqueness of the solution, the entire sequence $v_{\epsilon}$ converges to $v$.

\section{Homogenization of a fourth-order equation}

Our method also applies to fourth-order problems. Although systems of equations can be treated, for simplicity we focus on the case of a single 
equation, without loss of generality since there is no maximum principle for fourth-order elliptic equation. Let us introduce the following symmetric fourth-order operator

$$
\mathcal{A}^{\epsilon}=\operatorname{div} \operatorname{div}\left(\Theta\left(\frac{x}{\epsilon}\right) \nabla \nabla\right)-\frac{1}{\epsilon^{2}} \operatorname{div}\left(A\left(\frac{x}{\epsilon}\right) \nabla\right)+\frac{1}{\epsilon^{4}} c\left(\frac{x}{\epsilon}\right)+d\left(x, \frac{x}{\epsilon}\right),
$$

with periodic coefficients $\Theta(y)=\left\{\Theta_{i j k l}(y)\right\}, A(y)=\left\{A_{i j}(y)\right\}$ and $c(y)$ which are real periodic functions in $L^{\infty}\left(\mathbb{T}^{N}\right)$. Furthermore, $\Theta$ and $A$ are symmetric tensors, and $\Theta$ is uniformly elliptic. The locally periodic term $d(x, y)$ belongs to $L^{\infty}\left(\Omega ; C\left(\mathbb{T}^{N}\right)\right)$.

Under these assumptions the Bloch decomposition for $(112)$ is basically the same as that for second order operators. On the torus $\mathbb{T}^{N}$ we introduce the Bloch operators

$$
\begin{gathered}
\mathcal{A}(\theta) \psi(y)=e^{-2 i \pi y \cdot \theta} \mathcal{A} e^{-2 i \pi y \cdot \theta} \psi(y)= \\
\left(\nabla_{y}+2 i \pi \theta\right)\left(\nabla_{y}+2 i \pi \theta\right) \cdot\left(\Theta(y)\left(\nabla_{y}+2 i \pi \theta\right)\left(\nabla_{y}+2 i \pi \theta\right)\right) \psi(y)+ \\
-\left(\nabla_{y}+2 i \pi \theta\right) \cdot\left(A(y)\left(\nabla_{y}+2 i \pi \theta\right) \psi(y)+c(y) \psi(y)\right.
\end{gathered}
$$

with $\mathcal{A}=\operatorname{div} \operatorname{div}(\Theta(y) \nabla \nabla)-\operatorname{div}(A(y) \nabla)+c(y)$. Then, the Bloch spectral cell problem

$$
\mathcal{A}(\theta) \psi_{n}=\lambda_{n}(\theta) \psi_{n} \quad \text { in } \quad L^{2}\left(\mathbb{T}^{N}\right)
$$

has a discrete spectrum $\lambda_{1}(\theta) \leq \lambda_{2}(\theta) \leq \ldots \leq \lambda_{n}(\theta) \rightarrow+\infty$. Moreover, all the statements of Lemma 2.4 (and its rescaled version) remain valid.

It is quite natural to make assumption (97) which implies $\nabla_{\theta} \nabla_{\theta} \lambda_{1}\left(\theta_{0}\right)=$ 0 . For example, (97) is easily seen to be satisfied with $\theta_{0}=0$ if there are no zero and second order terms in (112), i.e. $A \equiv 0, c \equiv 0$.

We begin with the parabolic Cauchy problem

$$
\begin{cases}\frac{\partial u_{\epsilon}}{\partial t}+\mathcal{A}^{\epsilon} u_{\epsilon}=0 & \text { in } \Omega \times(0, T), \\ u_{\epsilon}=0, \quad \frac{\partial u_{\epsilon}}{\partial n}=0 & \text { on } \partial \Omega \times(0, T), \\ u_{\epsilon}(t=0, x)=u_{\epsilon}^{0}(x) & \text { in } \Omega .\end{cases}
$$

Theorem 9.1 Assume $\sqrt{97})$. Let $u_{\epsilon}(t, x)$ be a solution of $(113)$ with $\mathcal{A}^{\epsilon}$ given by 112), and $u_{\epsilon}^{0} \in L^{2}(\Omega)$ be an initial data of the form

$$
u_{\epsilon}^{0}(x)=\psi_{1}\left(\frac{x}{\epsilon}, \theta_{0}\right) e^{2 i \pi \frac{\theta_{0} \cdot x}{\epsilon}} v^{0}(x),
$$


with $v^{0} \in L^{2}(\Omega)$. Then $u_{\epsilon}$ can be written as

$$
u_{\epsilon}(t, x)=e^{-\frac{\lambda_{1}\left(\theta_{0}\right) t}{\epsilon^{4}}}\left(\psi_{1}\left(\frac{x}{\epsilon}, \theta_{0}\right) e^{2 i \pi \frac{\theta_{0} \cdot x}{\epsilon}} v_{\epsilon}(t, x)+r_{\epsilon}(t, x)\right),
$$

where the remainder term $r_{\epsilon}$ satisfies the relation

$$
\lim _{\epsilon \rightarrow 0}\left\|r_{\epsilon}\right\|_{L^{2}((0, T) \times \omega)}=0 \text { for any compact set } \omega \subset \mathbb{R}^{N},
$$

and $v_{\epsilon}$ converges weakly in $L^{2}\left((0, T) ; H^{2}(\Omega)\right)$ to the solution $v$ of

$$
\begin{cases}\frac{\partial v}{\partial t}+\mathcal{A}^{*} v=0 & \text { in } \Omega \times(0, T), \\ v=0, \quad \frac{\partial v}{\partial n}=0 & \text { on } \partial \Omega \times(0, T), \\ v(t=0, x)=v^{0}(x) & \text { in } \Omega,\end{cases}
$$

with the homogenized operator

$$
\mathcal{A}^{*}=\operatorname{div} \operatorname{div}\left(\Theta^{*} \nabla \nabla\right)+d^{*}(x)
$$

and $\Theta^{*}=\frac{1}{16 \pi^{4}} \nabla_{\theta} \nabla_{\theta} \nabla_{\theta} \nabla_{\theta} \lambda_{1}\left(\theta_{0}\right), d^{*}(x)=\int_{\mathbb{T}^{N}} d(x, y)\left|\psi_{1}\left(y, \theta_{0}\right)\right|^{2} d y$.

The proof of Theorem 9.1 is very similar to that of Theorem 8.2. Upon defining $\tilde{u}^{\epsilon}(t, x)=e^{\frac{\lambda_{1}\left(\theta_{0}\right) t}{\epsilon^{4}}} u^{\epsilon}(t, x)$, the a priori estimates are

$$
\left\|\tilde{u}^{\epsilon}\right\|_{L^{2}(\Omega)}+\epsilon\left\|\nabla \tilde{u}^{\epsilon}\right\|_{L^{2}(\Omega)^{N}}+\epsilon^{2}\left\|\nabla \nabla \tilde{u}^{\epsilon}\right\|_{L^{2}(\Omega)^{N^{2}}} \leq C,
$$

which, up to a subsequence, implies the following two-scale convergences for $w^{\epsilon}=e^{-2 i \pi x \cdot \theta_{0} / \epsilon} \tilde{u}^{\epsilon}(t, x)$

$w^{\epsilon} \stackrel{2 \mathrm{~s}}{\longrightarrow} v(t, x) \psi_{1}\left(y, \theta_{0}\right), \quad \epsilon \nabla w^{\epsilon} \stackrel{2 \mathrm{~s}}{\longrightarrow} v(t, x) \nabla_{y} \psi_{1}\left(y, \theta_{0}\right), \quad \epsilon^{2} \nabla \nabla w^{\epsilon} \stackrel{2 \mathrm{~s}}{\longrightarrow} v(t, x) \nabla_{y} \nabla_{y} \psi_{1}\left(y, \theta_{0}\right)$ where $v(t, x)$ is a limit point of a sequence $v^{\epsilon}$, bounded in $L^{2}\left((0, T) ; H^{2}\left(\mathbb{R}^{N}\right)\right)$, introduced in a variant of Lemmas 4.2 and 8.3 . Eventually, we use the same test function defined in (108). We safely leave the details to the reader.

We then study the Dirichlet spectral problem

$$
\mathcal{A}^{\epsilon} u_{n}^{\epsilon}=\lambda_{n}^{\epsilon} u_{n}^{\epsilon}, \quad u_{n}^{\epsilon} \in H_{0}^{2}(\Omega)
$$

stated in a bounded domain $\Omega \subset \mathbb{R}^{N}$, which, under the standing ellipticity assumptions, admits a discrete spectrum, $\lambda_{n}^{\epsilon} \rightarrow+\infty$ as $n \rightarrow+\infty$, with corresponding normalized eigenfunctions denoted by $u_{n}^{\epsilon}$. 
Theorem 9.2 Assume (97). Then for any $n \geq 1$

$$
\lambda_{n}^{\epsilon}=\frac{\lambda_{1}\left(\theta_{0}\right)}{\epsilon^{4}}+\mu_{n}+o(1) \quad \text { as } \epsilon \rightarrow 0
$$

and the corresponding eigenfunction $u_{n}^{\epsilon}(x)$ admits the representation

$$
u_{n}^{\epsilon}(x)=\psi_{1}\left(\frac{x}{\epsilon}, \theta_{0}\right) e^{2 i \pi \frac{\theta_{0} \cdot x}{\epsilon}} v_{n}^{\epsilon}(x)+r_{n}^{\epsilon}(x),
$$

where

$$
\lim _{\epsilon \rightarrow 0}\left\|r_{n}^{\epsilon}\right\|_{L^{2}(\Omega)}=0, \quad\left\|v_{n}^{\epsilon}\right\|_{H^{2}(\Omega)} \leq C, \quad \lim _{\epsilon \rightarrow 0}\left\|v_{n}^{\epsilon}\right\|_{L^{2}(\Omega)}=1,
$$

and the family $v_{n}^{\epsilon}$ is relatively compact in $L^{2}(\Omega)$. Moreover, any limit point $v_{n}^{0}$, as $\epsilon \rightarrow 0$, of the sequence $v_{n}^{\epsilon}$ is a normalized eigenfunction associated to the $n$-th eigenvalue $\mu_{n}$ of the scalar homogenized spectral problem

$$
\mathcal{A}^{*} v=\mu v \quad \text { in } \Omega, \quad v \in H_{0}^{2}(\Omega),
$$

with $\mathcal{A}^{*}$ defined by (114). If $\mu_{n}$ is a simple eigenvalue of the latter problem, the entire sequence $v_{n}^{\epsilon}$ converges to the homogenized eigenfunction $v_{n}$.

The proof is a combination of those of Theorems 3.6 and 8.2 . The crucial point is to obtain a uniform estimate for the energy $\left(\mathcal{A}^{\epsilon} u_{n}^{\epsilon}, u_{n}^{\epsilon}\right)$. To this end we use a test function of the type of (108).

Finally, for the hyperbolic system

$$
\begin{cases}\frac{\partial^{2} u_{\epsilon}}{\partial t^{2}}+\mathcal{A}^{\epsilon} u_{\epsilon}=0 & \text { in } \Omega \times(0, T), \\ u_{\epsilon}=0, \quad \frac{\partial u_{\epsilon}}{\partial n}=0 & \text { on } \partial \Omega \times(0, T), \\ u_{\epsilon}(0, x)=u_{\epsilon}^{0}(x) & \text { in } \Omega, \\ \frac{\partial u_{\epsilon}}{\partial t}(0, x)=u_{\epsilon}^{1}(x) & \text { in } \Omega,\end{cases}
$$

we obtain different homogenized limits according to the sign of $\lambda_{1}\left(\theta_{0}\right)$.

Theorem 9.3 Let $(97)$ be fulfilled, and assume that $\lambda_{1}\left(\theta_{0}\right)=0$ and the initial data are

$$
\begin{aligned}
& u_{\epsilon}^{0}(x)=\psi_{1}\left(\frac{x}{\epsilon}, \theta_{0}\right) e^{2 i \pi \frac{\theta_{0} \cdot x}{\epsilon}} v^{0}(x) \in H_{0}^{2}(\Omega), \\
& u_{\epsilon}^{1}(x)=\psi_{1}\left(\frac{x}{\epsilon}, \theta_{0}\right) e^{2 i \pi \frac{\theta_{0} \cdot x}{\epsilon}} v^{1}(x) \in L^{2}(\Omega),
\end{aligned}
$$


with $v^{0} \in H_{0}^{2}(\Omega)$ and $v^{1} \in L^{2}(\Omega)$. The solution of (117), with $\mathcal{A}^{\epsilon}$ given by (112), can be written as

$$
u_{\epsilon}(t, x)=\psi_{1}\left(\frac{x}{\epsilon}, \theta_{0}\right) e^{2 i \pi \frac{\theta_{0} \cdot x}{\epsilon}} v_{\epsilon}(t, x)+r_{\epsilon}(t, x),
$$

where the remainder term $r_{\epsilon}$ satisfies the relation

$$
\lim _{\epsilon \rightarrow 0}\left\|r_{\epsilon}\right\|_{L^{2}((0, T) \times \omega)}=0 \text { for any compact set } \omega \subset \mathbb{R}^{N},
$$

and $v_{\epsilon}$ converges weakly in $L^{2}\left((0, T) ; H^{2}(\Omega)\right)$ to the solution $v$ of

$$
\begin{cases}\frac{\partial^{2} v}{\partial t^{2}}+\mathcal{A}^{*} v=0 & \text { in } \Omega \times(0, T), \\ v=0, \quad \frac{\partial v}{\partial n}=0 & \text { on } \partial \Omega \times(0, T), \\ v(t=0, x)=v^{0}(x) & \text { in } \Omega, \\ \frac{\partial v}{\partial t}(t=0, x)=v^{1}(x) & \text { in } \Omega,\end{cases}
$$

with $\mathcal{A}^{*}$ defined by (114).

The proof is the same as that of Theorem 3.7. If $\lambda_{1}\left(\theta_{0}\right) \neq 0$, then we need to look at a different time scaling. Instead of (117), we now consider

$$
\begin{cases}\epsilon^{4} \frac{\partial^{2} u_{\epsilon}}{\partial t^{2}}+\mathcal{A}^{\epsilon} u_{\epsilon}=0 & \text { in } \Omega \times(0, T), \\ u_{\epsilon}=0, \quad \frac{\partial u_{\epsilon}}{\partial n}=0 & \text { on } \partial \Omega \times(0, T), \\ u_{\epsilon}(0, x)=u_{\epsilon}^{0}(x) & \text { in } \Omega, \\ \frac{\partial u_{\epsilon}}{\partial t}(0, x)=u_{\epsilon}^{1}(x) & \text { in } \Omega,\end{cases}
$$

Theorem 9.4 Let (97) be fulfilled, and assume that the initial data are

$$
u_{\epsilon}^{0}(x)=\psi_{1}\left(\frac{x}{\epsilon}, \theta_{0}\right) e^{2 i \pi \frac{\theta_{0} \cdot x}{\epsilon}} v^{0}(x) \in H_{0}^{2}(\Omega),
$$

with $v^{0} \in H_{0}^{2}(\Omega)$, and that $\epsilon^{4} u_{\epsilon}^{1}(x)$ is bounded in $L^{2}(\Omega)$ while $\epsilon^{4} \psi_{1}\left(\frac{x}{\epsilon}, \theta_{0}\right) u_{\epsilon}^{1}(x)$ converges weakly to 0 in $L^{2}(\Omega)$.

If $\lambda_{1}\left(\theta_{0}\right)<0$ the solution of (118) can be written as

$$
u_{\epsilon}(t, x)=e^{\frac{\sqrt{-\lambda_{1}\left(\theta_{0}\right) t}}{\epsilon^{4}}}\left(\psi_{1}\left(\frac{x}{\epsilon}, \theta_{0}\right) e^{2 i \pi \frac{\theta_{0} \cdot x}{\epsilon}} v_{\epsilon}(t, x)+r_{\epsilon}(t, x)\right),
$$


where $\lim _{\epsilon \rightarrow 0}\left\|r_{\epsilon}\right\|_{L^{2}((0, T) \times \omega)}=0$ for any compact set $\omega \subset \mathbb{R}^{N}$, and $v_{\epsilon}$ converges weakly in $L^{2}\left((0, T) ; H^{2}(\Omega)\right)$ to the solution $v$ of

$$
\begin{cases}2 \sqrt{-\lambda_{1}\left(\theta_{0}\right)} \frac{\partial v}{\partial t}+\mathcal{A}^{*} v=0 & \text { in } \Omega \times(0, T), \\ v=0, \quad \frac{\partial v}{\partial n}=0 & \text { on } \partial \Omega \times(0, T), \\ v(t=0, x)=\frac{1}{2} v^{0}(x) & \text { in } \Omega .\end{cases}
$$

If $\lambda_{1}\left(\theta_{0}\right)>0$ the solution of (118) satisifies

$$
u_{\epsilon}(t, x)=e^{i \frac{\sqrt{\lambda_{1}\left(\theta_{0}\right)} t}{\epsilon^{4}}} e^{2 i \pi \frac{\theta_{0} \cdot x}{\epsilon}} v_{\epsilon}(t, x),
$$

where $v_{\epsilon}$ two-scale converges to $\psi_{1}\left(y, \theta_{0}\right) v(t, x)$ and $v \in L^{2}\left((0, T) ; H_{0}^{2}(\Omega)\right)$ is the solution of

$$
\begin{cases}2 i \sqrt{\lambda_{1}\left(\theta_{0}\right)} \frac{\partial v}{\partial t}+\mathcal{A}^{*} v=0 & \text { in } \Omega \times(0, T), \\ v=0, \frac{\partial v}{\partial n}=0 & \text { on } \partial \Omega \times(0, T), \\ v(t=0, x)=\frac{1}{2} v^{0}(x) & \text { in } \Omega,\end{cases}
$$

with $\mathcal{A}^{*}$ defined by 114).

Again the proof is similar to those of Theorems 3.8 and 3.10 .

Assumption (97) is not the only possible one. In particular, it may happen that $\nabla_{\theta} \nabla_{\theta} \lambda_{1}\left(\theta_{0}\right)$ does not vanish at the minimum value $\theta_{0}$. Therefore, we now make assumption (9), i.e. $\nabla_{\theta} \nabla_{\theta} \lambda_{1}\left(\theta_{0}\right)$ is positive definite instead of (97).

Remark 9.5 We give an explicit example where (9) is satisfied rather than (97). Consider an arbitrary periodic, symmetric, uniformly elliptic, operator $\mathcal{B}$ of the form $\mathcal{B}=-\operatorname{div}_{y}\left(B(y) \nabla_{y}\right)+c(y)$ and its Bloch spectrum $\mu_{1}(\theta) \leq$ $\mu_{2}(\theta) \leq \ldots$ Adding, if necessary, a sufficiently large positive constant to $c$, we can assume that $\mu_{1}(\theta) \geq C>0$. Considering the relation

$$
\left(e^{-2 i \pi y \cdot \theta} \mathcal{B}^{2} e^{-2 i \pi y \cdot \theta}\right) \psi=\left(e^{-2 i \pi y \cdot \theta} \mathcal{B} e^{-2 i \pi y \cdot \theta}\right)\left(e^{-2 i \pi y \cdot \theta} \mathcal{B} e^{-2 i \pi y \cdot \theta}\right) \psi
$$

we conclude that the Bloch spectrum of the operator $\mathcal{A}=\mathcal{B}^{2}$ is $\left(\lambda_{n}(\theta)=\mu_{n}^{2}(\theta)\right)_{n>1}$.

According to Remark 2.1 the unique minimum point of $\mu_{1}$ is attained at $\theta_{0}=0$ and the matrix $\nabla_{\theta} \nabla_{\theta} \mu_{1}(0)$ is positive definite. Since $\mu_{1}(\theta)$ is strictly positive, the function $\lambda_{1}(\theta)=\mu_{1}^{2}(\theta)$ also has a unique minimum point at $\theta_{0}=0$ and its Hessian at 0 is positive definite. 
Under assumption (9) we need to change the scaling of $(112)$ and consider instead the new operator

$$
\mathcal{A}^{\epsilon}=\epsilon^{2} \operatorname{div} \operatorname{div}\left(\Theta\left(\frac{x}{\epsilon}\right) \nabla \nabla\right)-\operatorname{div}\left(A\left(\frac{x}{\epsilon}\right) \nabla\right)+\frac{1}{\epsilon^{2}} c\left(\frac{x}{\epsilon}\right)+d\left(x, \frac{x}{\epsilon}\right) .
$$

Then, the homogenization of the parabolic equation is given by a result similar to Theorem 3.2 .

Theorem 9.6 Assume (9). Let $u_{\epsilon}(t, x)$ be a solution of the parabolic equation (113) with $\mathcal{A}^{\epsilon}$ given by (119), and $u_{\epsilon}^{0} \in L^{2}(\Omega)$ be an initial data of the form

$$
u_{\epsilon}^{0}(x)=\psi_{1}\left(\frac{x}{\epsilon}, \theta_{0}\right) e^{2 \pi i \frac{\theta_{0} \cdot x}{\epsilon}} v^{0}(x)
$$

with $v^{0} \in L^{2}(\Omega)$. Then $u_{\epsilon}$ can be written as

$$
u_{\epsilon}(t, x)=e^{-\frac{\lambda_{1}\left(\theta_{0}\right) t}{\epsilon^{2}}}\left(\psi_{1}\left(\frac{x}{\epsilon}, \theta_{0}\right) e^{2 i \pi \frac{\theta_{0} \cdot x}{\epsilon}} v_{\epsilon}(t, x)+r_{\epsilon}(t, x)\right),
$$

where the remainder term $r_{\epsilon}$ satisfies

$$
\lim _{\epsilon \rightarrow 0}\left\|r_{\epsilon}\right\|_{L^{2}((0, T) \times \omega)}=0
$$

on any compact set $\omega \subset \mathbb{R}^{N}$, and $v_{\epsilon}$ converges weakly in $L^{2}\left((0, T) ; H^{1}(\Omega)\right)$ to the solution $v$ of the scalar homogenized problem

$$
\begin{cases}\frac{\partial v}{\partial t}-\operatorname{div}\left(A^{*} \nabla v\right)+d^{*}(x) v=0 & \text { in } \Omega \times(0, T), \\ v=0 & \text { on } \partial \Omega \times(0, T), \\ v(0, x)=v^{0}(x) & \text { in } \Omega,\end{cases}
$$

with $A^{*}=\frac{1}{4 \pi^{2}} \nabla_{\theta} \nabla_{\theta} \lambda_{1}\left(\theta_{0}\right)$ and $d^{*}(x)=\int_{\mathbb{T}^{N}} d(x, y)\left|\psi_{1}\left(y, \theta_{0}\right)\right|^{2} d y$.

The proof of Theorem 9.6 relies on the same test function than in the proof of Theorem 3.2. It should be noted that although $u^{\epsilon}(t, x)$ belongs to $L^{2}\left((0, T) ; H_{0}^{2}(\Omega)\right)$, the sequence $v^{\epsilon}$, defined in Theorem 9.6. is only bounded in $L^{2}\left((0, T) ; H^{1}\left(\mathbb{R}^{N}\right)\right)$, uniformly with respect to $\epsilon$. This is due to assumption (9) which allows us to prove Lemma 4.2 but not Lemma 8.3 .

Of course, similar results can be obtained for the spectral problem and for the hyperbolic equation: in both cases the homogenized operator is of second-order in space as in Theorem 9.6.

Acknowledgements. This work was partly done when A. Piatnitski and M. Vanninathan were visiting the Centre de Mathématiques Appliquées at Ecole Polytechnique. Y. Capdeboscq thanks L.C. Evans for bringing reference [24] to his attention. 


\section{References}

[1] J.H. Albert, Genericity of simple eigenvalues for elliptics pde's, Proc. A.M.S. 48:413-418 (1975).

[2] G. Allaire, Homogenization and two-scale convergence, SIAM J. Math. Anal. 23(6):1482-1518 (1992).

[3] G. Allaire, Dispersive limits in the homogenization of the wave equation, Internal report 510, CMAP, Ecole Polytechnique (2003).

[4] G. Allaire, Y. Capdeboscq, Homogenization of a spectral problem in neutronic multigroup diffusion, Comput. Methods Appl. Mech. Engrg. 187:91-117 (2000).

[5] G. Allaire, Y. Capdeboscq, Homogenization and localization for a 1-d eigenvalue problem in a periodic medium with an interface, Annali di Matematica 181, pp.247-282 (2002).

[6] G. Allaire, C. Conca, Bloch wave homogenization and spectral asymptotic analysis, J. Math. Pures et Appli. 77:153-208 (1998).

[7] G. Allaire, F. Malige, Analyse asymptotique spectrale d'un problème de diffusion neutronique, C. R. Acad. Sci. Paris Série I, t.324, pp.939-944 (1997).

[8] G. Allaire, A. Piatnitski, Uniform Spectral Asymptotics for Singularly Perturbed Locally Periodic Operators, Com. in PDE 27, pp.705-725 (2002).

[9] G. Allaire, A. Piatnitski, Homogenization of the Schrödinger equation and effective mass theorems, preprint (2003).

[10] P. Anselone, Collectively Compact Operator Approximation Theory and Applications to Integral Equations, Prentice-Hall, Englewood Cliffs, N.J., 1971.

[11] A. Bensoussan, J.-L. Lions, G. Papanicolaou, Asymptotic analysis for periodic structures, North-Holland, Amsterdam, 1978. 
[12] S. Brahim-Otsmane, G. Francfort, F. Murat, Correctors for the homogenization of the wave and heat equations, J. Math. Pures Appl. (9) 71:197-231 (1992).

[13] H. Brézis, Oprateurs maximaux monotones et semi-groupes de contractions dans les espaces de Hilbert, North-Holland, Amsterdam (1973).

[14] Y. Capdeboscq, Homogenization of a diffusion equation with drift, C. R. Acad. Sci. Paris Série I, t. 327:807-812 (1998).

[15] C. Castro, E. Zuazua, Low frequency asymptotic analysis of a string with rapidly oscillating density, SIAM J. Appl. Math. 60(4):1205-1233 (2000).

[16] C. Conca, R. Orive, M. Vanninathan, Bloch approximation in homogenization and applications, SIAM J. Math. Anal. 33:1166-1198 (2002).

[17] C. Conca, J. Planchard, M. Vanninathan, Fluids and periodic structures, RMA 38, J. Wiley \& Masson, Paris (1995).

[18] C. Conca, M. Vanninathan, Homogenization of periodic structures via Bloch decomposition, SIAM J. Appl. Math. 57:1639-1659 (1997).

[19] G. Geymonat, S. Müller, N. Triantafyllidis, Homogenization of nonlinearly elastic materials, microscopic bifurcation and macroscopic loss of rank-one convexity, Arch. Rational Mech. Anal. 122:231-290 (1993).

[20] V. V. Jikov, S. M. Kozlov, and O. A. Oleinik, Homogenization of Differential Operators and Integral Functionals. Springer Verlag (1994).

[21] S. Kozlov, Reducibility of quasiperiodic differential operators and averaging, Transc. Moscow Math. Soc., issue 2, 101-126 (1984).

[22] H.P. Myers, Introductory solid state physics, Taylor \& Francis, London (1990).

[23] G. Nguetseng, A general convergence result for a functional related to the theory of homogenization, SIAM J. Math. Anal. 20(3), pp. 608-623 (1989).

[24] F. Pedersen, Simple derivation of the effective-mass equation using a multiple-scale technique, Eur. J. Phys., 18, pp.43-45 (1997). 
[25] F. Poupaud, C. Ringhofer, Semi-classical limits in a crystal with exterior potentials and effective mass theorems, Comm. Partial Differential Equations, 21, no. 11-12, pp.1897-1918 (1996).

[26] M. Reed, B. Simon, Methods of modern mathematical physics, Academic Press, New York (1978).

[27] V. Siess, Homogenization of the simplified transport equation, $\mathrm{PhD}$ Thesis, Paris 6 University (2003).

[28] M. Vanninathan, Homogenization of eigenvalue problems in perforated domains, Proc. Indian Acad. Sci. Math. Sci., 90:239-271 (1981). 